\title{
ON THE SIEGEL-WEIL FORMULA OVER FUNCTION FIELDS
}

\author{
FU-TSUN WEI
}

\begin{abstract}
The aim of this article is to prove the Siegel-Weil formula over function fields for the dual reductive pair $\left(\mathrm{Sp}_{n}, \mathrm{O}(V)\right)$, where $\mathrm{Sp}_{n}$ is the symplectic group of degree $2 n$ and $\left(V, Q_{V}\right)$ is an anisotropic quadratic space with even dimension. This is a function field analogue of Kudla and Rallis' result. By this formula, the theta series is identified with the special value of the Siegel-Eisenstein series on $\mathrm{Sp}_{n}$ at a critical point.
\end{abstract}

\section{INTRODUCTION}

In the number field case, the Siegel-Weil formula, discovered by Siegel [16] and considerably extended by Weil ([18] and [19]) in the representation theoretical language, connects special values of Eisenstein series with theta series from quadratic spaces. In [19], Weil assumed the critical point in question lies in the absolute convergence of the Eisenstein series. After Weil, Kudla and Rallis ([9], [10], and [15]) explored the analytic behavior of the meromorphic continuation of the Eisenstein series, and extended Weil's result to the case beyond the convergence range for the dual reductive pair $\left(\mathrm{Sp}_{n}, \mathrm{O}(V)\right)$. Here $\left(V, Q_{V}\right)$ is a non-degenerate quadratic space with even dimension, $\mathrm{O}(V)$ is the associated orthogonal group, and $\mathrm{Sp}_{n}$ is the symplectic group of degree $2 n$. Many extensions and variations of this formula are studied in the number field case, and have applications to the special values of automorphic $L$-functions. However, there is a lack of knowledge about this formula in the function field context, except, Harris [4] dealt with $\left(\mathrm{SL}_{2}, \mathrm{O}(V)\right)$ (resp. $\left(\widetilde{\mathrm{SL}_{2}}, \mathrm{O}(V)\right)$, where $\widetilde{\mathrm{SL}_{2}}$ is the metaplectic cover of $\mathrm{SL}_{2}$ ) when the dimension of $V$ is even (resp. odd) and larger than 4. Our purpose in this article is to show a function field analogue of the Siegel-Weil formula for the dual reductive pair $\left(\mathrm{Sp}_{n}, \mathrm{O}(V)\right)$ where $V$ is an anisotropic quadratic space with even dimension, in order to complete the author's work ([2], [3], and [17]) on the central critical values of $L$-functions coming from "Drinfeld type" autumorphic forms.

Let $k$ be a global function field with odd characteristic. Let $\left(V, Q_{V}\right)$ be an anisotropic quadratic space over $k$ with even dimension. Take a Schwartz function $\varphi \in S\left(V\left(\mathbb{A}_{k}\right)^{n}\right)$, where $\mathbb{A}_{k}$ is the adele ring of $k$, the associated theta series on $\operatorname{Sp}_{n}\left(\mathbb{A}_{k}\right) \times \mathrm{O}(V)\left(\mathbb{A}_{k}\right)$ is defined by

$$
\theta(g, h, \varphi):=\sum_{x \in V(k)}(\omega(g, h) \varphi)(x), \quad \forall(g, h) \in \operatorname{Sp}_{n}\left(\mathbb{A}_{k}\right) \times \mathrm{O}(V)\left(\mathbb{A}_{k}\right) .
$$

Here $\omega$ is the Weil representation of $\operatorname{Sp}_{n}\left(\mathbb{A}_{k}\right) \times \mathrm{O}(V)\left(\mathbb{A}_{k}\right)$ on the Schwartz space $S\left(V\left(\mathbb{A}_{k}\right)^{n}\right)$ (cf. Section 1.3). Since $V$ is anisotropic, the following integral is well-defined:

$$
I^{n}(g, \varphi):=\int_{\mathrm{O}(V)(k) \backslash \mathrm{O}(V)\left(\mathbb{A}_{k}\right)} \theta(g, h, \varphi) d h .
$$

The measure $d h$ is induced from the Haar measure on $\mathrm{O}(V)\left(\mathbb{A}_{k}\right)$ normalized so that the volume of $\mathrm{O}(V)(k) \backslash \mathrm{O}(V)\left(\mathbb{A}_{k}\right)$ is 1 . Then the main theorem of this article is:

2000 Mathematics Subject Classification. 11F27, 11F55, 11M36, 11R58.

Key words and phrases. Function field, Theta series, Eisenstein series, Automorphic form. 
Theorem 0.1. For $\varphi \in S\left(V\left(\mathbb{A}_{k}\right)^{n}\right)$ and $g \in \operatorname{Sp}_{n}\left(\mathbb{A}_{k}\right)$,

$$
E\left(g, s_{(n)}, \Phi_{\varphi}\right)=\epsilon(n) \cdot I^{n}(g, \varphi) .
$$

Here:

(i) $E\left(g, s, \Phi_{\varphi}\right)$ is the Siegel-Eisenstein series on $\operatorname{Sp}_{n}\left(\mathbb{A}_{k}\right)$ associated to the Siegel section $\Phi_{\varphi}$ (cf. Section 3);

(ii) $s_{(n)}=\operatorname{dim}(V) / 2-(n+1) / 2$; and

$$
\epsilon(n)= \begin{cases}1 & \text { if } \operatorname{dim}(V)>n+1 \\ 2 & \text { if } \operatorname{dim}(V) \leq n+1\end{cases}
$$

Note that $s_{(n)}$ could be out of the convergence region of the Siegel-Eisenstein series: $\operatorname{Re}(s)>(n+1) / 2$. In particular, the functional equation of the Eisenstein series relates the values at $s$ and $-s$, which means that $s_{(n)}=0$ is the central critical point of the Eisenstein series when $\operatorname{dim}(V)=n+1$. This gives an application to the central critical values of automorphic $L$-functions. In concrete terms, we express the $L$-function in which we are interested by an integral representation involving the Siegel-Eisenstein series. Then at $s=s_{(n)}$, the theta series $I^{n}(g, \varphi)$ provides a plenty of arithmetic information and leads us to explicit formulas for the special values of $L$-functions in question.

The strategy of the proof for Theorem 0.1 follows from [9]. The first step is to show that $I^{n}(g, \varphi)$ and $E\left(g, s, \Phi_{\varphi}\right)$ are both concentrated on the standard Borel subgroup of $\operatorname{Sp}_{n}$ (Proposition 2.3 and 3.2). Then we review the meromorphic continuation of the Eisenstein series, and make sure that $E\left(g, s, \Phi_{\varphi}\right)$ is holomorphic at $s_{(n)}$. Finally, we prove the equality of the constant term of $I^{n}(g, \varphi)$ and $E\left(g, s_{(n)}, \Phi_{\varphi}\right)$ along the Siegel-parabolic subgroup. Therefore $E\left(g, s_{(n)}, \Phi_{\varphi}\right)-\epsilon(n) I^{n}(g, \varphi)$ must be a cusp form on $\operatorname{Sp}_{n}\left(\mathbb{A}_{k}\right)$ which is orthogonal to all the cusp forms on $\operatorname{Sp}_{n}\left(\mathbb{A}_{k}\right)$. This assures the result. It is worth pointing out that the dimension of $V$ must be 2 or 4 by Hasse-Minkowski principle. In particular:

- when $\operatorname{dim}(V)=2,\left(V, Q_{V}\right) \cong\left(F, \alpha \cdot \operatorname{Nr}_{F / k}\right)$ for $\alpha \in k^{\times}$where $F / k$ is a quadratic extension and $\mathrm{Nr}_{F / k}$ is the norm form on $F / k$;

- when $\operatorname{dim}(V)=4$ and the discriminant of $V$ is a square in $k,\left(V, Q_{V}\right) \cong\left(D, \operatorname{Nr}_{D / k}\right)$ where $D$ is a division quaternion algebra over $k$ and $\mathrm{Nr}_{D / k}$ is the reduced norm form on $D / k$;

- when $\operatorname{dim}(V)=4$ and the discriminant $d$ of $V$ is a non-square in $k$,

$$
\left(V, Q_{V}\right) \cong\left(F_{d}^{o} \oplus D^{o}, \alpha \cdot\left(\mathrm{Nr}_{F^{\circ} / k} \oplus \mathrm{Nr}_{D^{\circ} / k}\right)\right)
$$

for $\alpha \in k^{\times}$where $F_{d}=k(\sqrt{d}), D$ is a division quaternion algebra over $k$ such that $F_{d}$ cannot embed into $D$, and

$$
F_{d}^{o}\left(\text { resp. } D^{o}\right)=\left\{b \in F_{d}(\text { resp. } D): \operatorname{Tr}(b)=0\right\} .
$$

This observation simplifies the proof. However, there are several techniques used in [9] which were not verified in the function field case. Therefore for the sake of completeness, some further discussions are sought in the appendices, including Fourier coefficients of theta series, the Jacquet module of the Schwartz space, and Maass-Jacquet-Shalika Eisenstein series on $\mathrm{GL}_{n}$. We point out that in the number field case, Kudla-Rallis use a differential operator (introduced by Maass) at one archimedean place to obtain the continuation and also the functional equation of the Maass-Jacquet-Shalika Eisenstein series. Our approach is to write down directly the explicit form of the meromorphic continuation, and the functional equation shows up accordingly.

The structure of this article is organized as follows. We set up basic notations in Section 1.1 and 1.2, and recall the Weil representation of $\operatorname{Sp}_{n}\left(\mathbb{A}_{k}\right) \times \mathrm{O}(V)\left(\mathbb{A}_{k}\right)$ on the Schwartz space 
$S\left(V\left(\mathbb{A}_{k}\right)^{n}\right)$ in Section 1.3. In Section 2, we introduce the theta series $I^{n}(\cdot, \varphi)$, and show that it is concentrated on the standard Borel subgroup of $\mathrm{Sp}_{n}$. In Section 3, we investigate the analytic behavior of Siegel-Eisenstein series by studying the constant terms along standard parabolic subgroups. Also shown in Section 3 is that the Eisenstein series are concentrated on the Borel subgroup of $\mathrm{Sp}_{n}$. We recall in Section 4 the meromorphic continuation of the intertwining operators on Siegel sections, and deduce in Section 5 the holomorphic property of Siegel-Eisenstein series at $s_{(n)}$ by using Maass-Jacquet-Shalika Eisenstein series on $\mathrm{GL}_{n}$. Finally, we show the Siegel-Weil formula for the case when $\operatorname{dim}(V)=n+1$ in $\operatorname{Section} 6.1$, and prove the other cases by a finite induction process in Section 6.2 and 6.3. In Appendix A, we follow Rallis [15] to study the non-singular Fourier coefficients of $I^{n}(g, \varphi)$ and $E\left(g, s, \Phi_{\varphi}\right)$. Appendix B is a review of the Jordan structure of the Jacquet module of the Schwartz space $S\left(V\left(k_{v}\right)^{n}\right.$ ) (where $k_{v}$ is the completion of $k$ at a place $v$ ). Finally, we show the meromorphic continuation and functional equation of the Maass-Jacquet-Shalika Eisenstein series on $\mathrm{GL}_{n}$ in Appendix C.

\section{Preliminary}

1.1. Basic setting. Let $k$ be a global function field with finite constant field $\mathbb{F}_{q}$, i.e. $k$ is a finitely generated field extension of transcendence degree one over $\mathbb{F}_{q}$ and $\mathbb{F}_{q}$ is algebraically closed in $k$. In this article we always assume that $q$ is odd. For each place $v$ of $k$, the completion of $k$ at $v$ is denoted by $k_{v}$, and $O_{v}$ is the valuation ring in $k_{v}$. Take a uniformizer $\pi_{v}$ in $O_{v}$. We set $\mathbb{F}_{v}:=O_{v} / \pi_{v} O_{v}$, the residue field at $v$. The cardinality of $\mathbb{F}_{v}$ is denoted by $q_{v}$. For each $\alpha \in k_{v}$,

$$
|\alpha|_{v}:=q_{v}^{-\operatorname{ord}_{v}(\alpha)} \text {. }
$$

The adele ring of $k$ is denoted by $\mathbb{A}_{k}$. We let $O_{\mathbb{A}_{k}}:=\prod_{v} O_{v}$, the maximal compact subring of $\mathbb{A}_{k}$. For any element $\alpha=\left(\alpha_{v}\right)_{v} \in \mathbb{A}_{k}^{\times}$, the norm $|\alpha|_{\mathbb{A}_{k}}$ is defined to be

$$
|\alpha|_{\mathbb{A}_{k}}:=\prod_{v}\left|\alpha_{v}\right|_{v}
$$

We fix a non-trivial additive character $\psi=\otimes_{v} \psi_{v}: \mathbb{A}_{k} \rightarrow \mathbb{C}^{\times}$which is trivial on $k$ (here $\psi_{v}\left(x_{v}\right):=\psi\left(0, \ldots, 0, x_{v}, 0, \ldots\right)$, for all $x_{v}$ in $\left.k_{v}\right)$.

1.2. The symplectic group $\mathrm{Sp}_{n}$. For a positive integer $n$, let

$$
\mathrm{Sp}_{n}=\left\{g \in \mathrm{GL}_{2 n} \mid{ }^{t} g\left(\begin{array}{cc}
0 & I_{n} \\
-I_{n} & 0
\end{array}\right) g=\left(\begin{array}{cc}
0 & I_{n} \\
-I_{n} & 0
\end{array}\right)\right\} .
$$

We view $\mathrm{Sp}_{n}$ as an affine algebraic group over $k$. Let $B_{n}=T_{n} \cdot U_{n}$ be the standard Borel subgroup of $\mathrm{Sp}_{n}$ where

$$
T_{n}=\left\{\left(\begin{array}{cc}
a & 0 \\
0 & { }^{t} a^{-1}
\end{array}\right) \mid a=\left(\begin{array}{ccc}
t_{1} & & \\
& \ddots & \\
& & t_{n}
\end{array}\right)\left(=: \operatorname{diag}\left(t_{1}, \ldots, t_{n}\right)\right), t_{i} \in \mathbb{G}_{m}\right\}
$$

and

$$
U_{n}=\left\{\left(\begin{array}{cc}
a & * \\
0 & { }^{t} a^{-1}
\end{array}\right) \in \operatorname{Sp}_{n} \mid a=\left(\begin{array}{ccc}
1 & & * \\
& \ddots & \\
0 & & 1
\end{array}\right) \in \mathrm{GL}_{n}\right\} .
$$

A parabolic subgroup $P$ of $\mathrm{Sp}_{n}$ is called standard if $B_{n}$ is a subgroup of $P$. The Siegel-parabolic subgroup $P_{n}$ is equal to $M_{n} \cdot N_{n}$, where

$$
M_{n}:=\left\{\mathfrak{m}(a)=\left(\begin{array}{cc}
a & 0 \\
0 & { }^{t} a^{-1}
\end{array}\right) \mid a \in \mathrm{GL}_{n}\right\}
$$


and

Let

$$
N_{n}:=\left\{\mathfrak{n}(b)=\left(\begin{array}{cc}
I_{n} & b \\
0 & I_{n}
\end{array}\right) \mid b={ }^{t} b \in \operatorname{Mat}_{n}\right\} .
$$

$$
\operatorname{Sym}_{n}:=\left\{b={ }^{t} b \in \operatorname{Mat}_{n}\right\} .
$$

Note that $\mathrm{GL}_{n}$ and $\mathrm{Sym}_{n}$ are isomorphic to $M_{n}$ and $N_{n}$ by the map $\mathfrak{m}$ and $\mathfrak{n}$ respectively. For $0<r<n$ we let $P_{r}=M_{r} N_{r}$ be the maximal proper parabolic subgroup of $\mathrm{Sp}_{n}$ where

$$
\begin{gathered}
N_{r}=\left\{\left(\begin{array}{cccc}
I_{r} & x & y & z \\
0 & I_{n-r} & { }^{t} z & 0 \\
0 & 0 & I_{r} & 0 \\
0 & 0 & -{ }^{t} x & I_{n-r}
\end{array}\right) \in \mathrm{Sp}_{n}\right\}, \\
M_{r}=\left\{\left(\begin{array}{cccc}
\alpha & 0 & 0 & 0 \\
0 & a & 0 & b \\
0 & 0 & { }^{t} \alpha^{-1} & 0 \\
0 & c & 0 & d
\end{array}\right) \mid \alpha \in \mathrm{GL}_{r},\left(\begin{array}{ll}
a & b \\
c & d
\end{array}\right) \in \mathrm{Sp}_{n-r}\right\} .
\end{gathered}
$$

The above description of $M_{r}$ gives us a natural isomorphism between $M_{r}$ and $\mathrm{GL}_{r} \times \mathrm{Sp}_{n-r}$.

Let $N_{\mathrm{Sp}_{n}}\left(T_{n}\right)$ be the normalizer of $T_{n}$ in $\mathrm{Sp}_{n}$. The Wyel group $N_{\mathrm{Sp}_{n}}\left(T_{n}\right) / T_{n}$ is denoted by $W_{\mathrm{Sp}_{n}}$. We shall use the same symbol for an element of $N_{\mathrm{Sp}_{n}}\left(T_{n}\right)$ and its image in $W_{\mathrm{Sp}_{n}}$. The Bruhat decomposition says that

$$
\mathrm{Sp}_{n}=\coprod_{w \in W_{\mathrm{Sp}_{n}}} B_{n} w B_{n}
$$

Let $X\left(T_{n}\right)$ be the group of (algebraic) characters on $T_{n}$. Then $X\left(T_{n}\right)=\oplus_{i=1}^{n} \mathbb{Z} x_{i}$, where $x_{i}$ is the character on $T_{n}$ satisfying

$$
x_{i}\left(\operatorname{diag}\left(t_{1}, \ldots, t_{n}\right)\right)=t_{i} .
$$

We define an $\mathbb{R}$-bilinear form $<\cdot, \cdot>$ on $X\left(T_{n}\right) \otimes_{\mathbb{Z}} \mathbb{R}$ by setting

$$
<x_{i}, x_{j}>= \begin{cases}1 & \text { if } i=j, \\ 0 & \text { otherwise }\end{cases}
$$

and extending bilinearly. The left action of $W_{\mathrm{Sp}_{n}}$ on $X\left(T_{n}\right) \otimes_{\mathbb{Z}} \mathbb{R}$ (induced by conjugation on $\left.T_{n}\right)$ is orthogonal with respect to $\langle\cdot, \cdot\rangle$, i.e.,

$$
<w x, w y>=<x, y>\text { for all } w \in W_{\mathrm{Sp}_{n}} \text { and } x, y \in X\left(T_{n}\right) \otimes_{\mathbb{Z}} \mathbb{R} .
$$

The set of roots of $\mathrm{Sp}_{n}$ with respect to $T_{n}$ is denoted by $\Delta_{n}$. For each root $\alpha \in \Delta_{n}$, let $N_{\alpha}$ be the unipotent subgroup of $\mathrm{Sp}_{n}$ associated to $\alpha$, and the reflection associated to $\alpha$ is denoted by $w_{\alpha}$. We let $\Delta_{n}^{+}$be the set of positive roots (with respect to $B_{n}$ ), and the simple roots are

$$
\begin{aligned}
\alpha_{i} & =x_{i}-x_{i+1}, 1 \leq i<n, \\
\alpha_{n} & =2 x_{n} .
\end{aligned}
$$

It is known that the Wyel group $W_{\mathrm{Sp}_{n}}$ is generated by $w_{\alpha_{i}}$.

1.3. The Weil representation of $\mathrm{Sp}_{n} \times \mathrm{O}(V)$. Let $\left(V, Q_{V}\right)$ be an anisotropic quadratic space over $k$ which has even dimension. By Hasse-Minkowski principle (cf. Theorem 2.12 and Section 3.1 in [11]), the dimension of $V$ must be 2 or 4 . Set

$$
<x, y>_{V}:=Q_{V}(x+y)-Q_{V}(x)-Q_{V}(y), \quad \forall x, y \in V,
$$

the bilinear form associated to $Q_{V}$. The orthogonal group of $V$ is denoted by $\mathrm{O}(V)$, i.e.

$$
\mathrm{O}(V)=\left\{h \in \mathrm{GL}(V) \mid Q_{V}(h x)=Q_{V}(x), \quad \forall x \in V\right\} .
$$

Here we view $\mathrm{O}(V)$ as an affine algebraic group over $k$. 
For each place $v$ of $k$, we have fixed an additive character $\psi_{v}$ on $k_{v}$ in Section 1.1. Let $V\left(k_{v}\right):=V \otimes_{k} k_{v}$ and let $S\left(V\left(k_{v}\right)^{n}\right)$ be the space of $S$ chwartz functions on $V\left(k_{v}\right)^{n}$, i.e. the space of functions on $V\left(k_{v}\right)$ which are locally constant and compactly supported. The (local) Weil representation $\omega_{v}\left(=\omega_{v, \psi_{v}}\right)$ of $\operatorname{Sp}_{n}\left(k_{v}\right) \times O(V)\left(k_{v}\right)$ on $S\left(V\left(k_{v}\right)^{n}\right)$ is determined by the following: for every $\varphi_{v} \in S\left(V\left(k_{v}\right)^{n}\right)$ and $x \in V\left(k_{v}\right)^{n}$,

$$
\begin{aligned}
\left(\omega_{v}(h) \varphi_{v}\right)(x) & :=\varphi_{v}\left(h^{-1} x_{1}, \ldots, h^{-1} x_{n}\right), \forall h \in \mathrm{O}(V)\left(k_{v}\right), ; \\
\left(\omega_{v}\left(\begin{array}{cc}
a & 0 \\
0 & { }^{t} a^{-1}
\end{array}\right) \varphi_{v}\right)(x) & :=\chi_{V, v}(\operatorname{det} a)|\operatorname{det} a|_{v}^{\frac{\operatorname{dim}(V)}{2}} \cdot \varphi_{v}(x \cdot a), \forall a \in \mathrm{GL}_{n}\left(k_{v}\right) ; \\
\left(\omega_{v}\left(\begin{array}{cc}
I_{n} & b \\
0 & I_{n}
\end{array}\right) \varphi_{v}\right)(x) & :=\psi_{v}\left(\operatorname{Trace}\left(b \cdot Q_{V}^{(n)}(x)\right)\right) \cdot \varphi_{v}(x), \forall b \in \operatorname{Sym}_{n}\left(k_{v}\right) ; \\
\left(\omega_{v}\left(\begin{array}{cc}
0 & I_{n} \\
-I_{n} & 0
\end{array}\right) \varphi_{v}\right)(x) & :=\varepsilon_{v}(V)^{n} \cdot \widehat{\varphi}_{v}(x) .
\end{aligned}
$$

Here:

- $\chi_{V, v}: k_{v}^{\times} \rightarrow\{ \pm 1\}$ is the quadratic character associated to $V$ at $v$, i.e.

$$
\chi_{V, v}(\alpha):=\left(\alpha,(-1)^{\frac{\operatorname{dim}(V)}{2}} \operatorname{det}(V)\right)_{v},
$$

where $(\cdot, \cdot)_{v}: k_{v}^{\times} \times k_{v}^{\times} \rightarrow\{ \pm 1\}$ is the Hilbert quadratic symbol and

$$
\operatorname{det}(V):=\operatorname{det}\left(\left(\frac{1}{2}<x_{i}, x_{j}>_{V}\right)_{1 \leq i, j \leq \operatorname{dim}(V)}\right) \in k^{\times} /\left(k^{\times}\right)^{2}
$$

for any $k$-basis $\left\{x_{1}, \ldots, x_{\operatorname{dim}(V)}\right\}$ of $V$.

- $Q_{V}^{(n)}: V^{n} \rightarrow \operatorname{Sym}_{n}$ is the moment map, i.e. for any $x=\left(x_{1}, \ldots, x_{n}\right) \in V^{n}$,

$$
Q_{V}^{(n)}(x)=\left(\frac{1}{2}<x_{i}, x_{j}>_{V}\right)_{1 \leq i, j \leq n} .
$$

- $\widehat{\varphi}_{v}$ is the Fourier transform of $\varphi_{v}$ (with respect to $\left.\psi_{v}\right)$ :

$$
\widehat{\varphi}_{v}(x):=\int_{V\left(k_{v}\right)^{n}} \varphi_{v}(y) \cdot \psi_{v}\left(\sum_{i=1}^{n}<x_{i}, y_{i}>_{V}\right) d y, \quad \forall x=\left(x_{1}, \ldots, x_{n}\right) \in V\left(k_{v}\right)^{n} \text {. }
$$

The Haar measure $d y=d y_{1} \cdots d y_{n}$ is chosen to be self dual, i.e.

$$
\widehat{\widehat{\varphi}}_{v}(x)=\varphi_{v}(-x), \quad \forall x \in V\left(k_{v}\right)^{n} \text {. }
$$

- $\varepsilon_{v}(V)$ is the Weil index of $V$ at $v$, i.e.

$$
\varepsilon_{v}(V):=\int_{L_{v}} \psi_{v}\left(Q_{V}(x)\right) d x
$$

for any sufficiently large $O_{v}$-lattice $L_{v}$ in $V\left(k_{v}\right)$. The Haar measure $d x$ is also chosen to be self dual.

We denote $\chi_{V}$ to be the character $\otimes_{v} \chi_{V, v}: k^{\times} \backslash \mathbb{A}_{k}^{\times} \rightarrow\{ \pm 1\}$.

Fix an arbitrary $k$-basis $\Lambda$ of $V$. For each place $v$ of $k$, let $\varphi_{v}^{0}$ be the characteristic function $\Lambda_{v}^{n} \subset V\left(k_{v}\right)^{n}$, where $\Lambda_{v} \subset V\left(k_{v}\right)$ is the $O_{v}$-lattice generated by the elements in $\Lambda$. Then for almost all places $v$ of $k$, it is known that

$$
\omega_{v}\left(\kappa_{v}, \kappa_{v}^{\prime}\right) \varphi_{v}^{0}=\varphi_{v}^{0} \forall\left(\kappa_{v}, \kappa_{v}^{\prime}\right) \in \operatorname{Sp}_{n}\left(O_{v}\right) \times \mathrm{O}(V)\left(O_{v}\right) .
$$

Let $V\left(\mathbb{A}_{k}\right):=V \otimes_{k} \mathbb{A}_{k}$ and let $S\left(V\left(\mathbb{A}_{k}\right)\right)$ be the space of Schwartz functions on $V\left(\mathbb{A}_{k}\right)$. Viewing $S\left(V\left(\mathbb{A}_{k}\right)^{n}\right)$ as the restricted tensor product $\otimes_{v}^{\prime} S\left(V\left(k_{v}\right)^{n}\right)$ with respect to $\left\{\varphi_{v}^{0}\right\}_{v}$, we have the (global) Weil representation $\omega=\otimes_{v} \omega_{v}$ of $\operatorname{Sp}_{n}\left(\mathbb{A}_{k}\right) \times \mathrm{O}(V)\left(\mathbb{A}_{k}\right)$ on the space $S\left(V\left(\mathbb{A}_{k}\right)^{n}\right)$ : for every $\varphi=\otimes_{v} \varphi_{v} \in S\left(V\left(\mathbb{A}_{k}\right)^{n}\right)$ and $(g, h)=\left(g_{v}, h_{v}\right)_{v}$ in $\operatorname{Sp}_{n}\left(\mathbb{A}_{k}\right) \times \mathrm{O}(V)\left(\mathbb{A}_{k}\right)$,

$$
\omega(g, h) \varphi:=\otimes_{v} \omega_{v}\left(g_{v}, h_{v}\right) \varphi_{v} .
$$




\section{THETA SERIES}

Take a Schwartz function $\varphi \in S\left(V\left(\mathbb{A}_{k}\right)^{n}\right)$. For $(g, h) \in \operatorname{Sp}_{n}\left(\mathbb{A}_{k}\right) \times \mathrm{O}(V)\left(\mathbb{A}_{k}\right)$, the theta series

$$
\theta(g, h, \varphi):=\sum_{x \in V(k)^{n}}(\omega(g) \varphi)\left(h^{-1} x\right),
$$

as a function on $\operatorname{Sp}_{n}\left(\mathbb{A}_{k}\right) \times \mathrm{O}(V)\left(\mathbb{A}_{k}\right)$, is left $\operatorname{Sp}_{n}(k) \times \mathrm{O}(V)(k)$-invariant. We define

$$
I^{n}(g, \varphi):=\int_{\mathrm{O}(V)(k) \backslash \mathrm{O}(V)\left(\mathbb{A}_{k}\right)} \theta(g, h, \varphi) d h .
$$

This integral is absolutely convergent, as $\mathrm{O}(V)(k) \backslash \mathrm{O}(V)\left(\mathbb{A}_{k}\right)$ is compact. The measure $d h$ is induced from the Haar measure on $\mathrm{O}(V)\left(\mathbb{A}_{k}\right)$ which is normalized so that the volume of $\mathrm{O}(V)(k) \backslash \mathrm{O}(V)\left(\mathbb{A}_{k}\right)$ is 1 .

Let $P$ be a standard parabolic subgroup of $\mathrm{Sp}_{n}$. Write $P=M \cdot N$, where $N$ is the unipotent radical of $P$ and $M$ is its standard Levi subgroup. Define

$$
I_{P}^{n}(g, \varphi):=\int_{N(k) \backslash N\left(\mathbb{A}_{k}\right)} I^{n}(n g, \varphi) d n, \quad \forall g \in \operatorname{Sp}_{n}\left(\mathbb{A}_{k}\right),
$$

where the measure $d n$ on $N(k) \backslash N\left(\mathbb{A}_{k}\right)$ is chosen so that the total mass is 1 . It is clear that

Lemma 2.1. $I_{P_{n}}^{n}(g, \varphi)=(\omega(g) \varphi)(0)$.

Let $Z_{M}$ denotes the center of $M$, which is contained in $T_{n}$.

Lemma 2.2. For every standard parabolic subgroup $P$ of $\operatorname{Sp}_{n}$, there exists a character $\nu_{P}$ on $Z_{M}(k) \backslash Z_{M}\left(\mathbb{A}_{k}\right)$ such that for every $z \in Z_{M}\left(\mathbb{A}_{k}\right), g \in \operatorname{Sp}_{n}\left(\mathbb{A}_{k}\right), \varphi \in S\left(V\left(\mathbb{A}_{k}\right)^{n}\right)$,

$$
I_{P}^{n}(z g, \varphi)=\nu_{P}(z) I_{P}^{n}(g, \varphi) .
$$

Proof. It is clear when $P=\mathrm{Sp}_{n}$, as the center of $\mathrm{Sp}_{n}$ is $\{ \pm 1\}$. Next, we consider the case when $P=P_{r}, 0<r \leq n$. Then $M_{r} \cong \mathrm{GL}_{r} \times \mathrm{Sp}_{n-r}$, and

$$
I_{P_{r}}^{n}(g, \varphi)=\int_{\mathrm{O}(V)(k) \backslash \mathrm{O}(V)\left(\mathbb{A}_{k}\right)} \sum_{x \in V(k)^{n-r}} \omega(g) \varphi\left(0, h^{-1} x\right) d h .
$$

Therefore for $m=\left(a, g^{\prime}\right) \in \mathrm{GL}_{r}\left(\mathbb{A}_{k}\right) \times \operatorname{Sp}_{n-r}\left(\mathbb{A}_{k}\right) \cong M_{r}\left(\mathbb{A}_{k}\right)$, we get

$$
I_{P}^{n}(m g, \varphi)=\chi_{V}(\operatorname{det} a)|\operatorname{det} a|_{\mathbb{A}_{k}}^{\frac{\operatorname{dim} V}{2}} I^{n-r}\left(g^{\prime}, \widetilde{\varphi}\right),
$$

where $\widetilde{\varphi} \in S\left(V\left(\mathbb{A}_{k}\right)^{n-r}\right)$ is defined by

$$
\widetilde{\varphi}(x):=\omega(g) \varphi(0, x) .
$$

This assures the result for $P=P_{r}$.

In general, we can assume that $P$ is contained in $P_{r}, 0<r \leq n$, and the Levi subgroup $M$ of $P$ is isomorphic to

$$
\mathrm{GL}_{r_{1}} \times \cdots \times \mathrm{GL}_{r_{\ell}} \times \mathrm{Sp}_{n-r}
$$

where $r_{1}+\cdots+r_{\ell}=r$. Note that $N=U \cdot N_{r}$ where $U$ is a unipotent subgroup of $M_{r}$. Therefore

$$
I_{P}^{n}(g, \varphi)=I_{P_{r}}^{n}(g, \varphi),
$$

which completes the proof immediately.

The next proposition shows that $I^{n}(g, \varphi)$ is concentrated on the Borel subgroup $B_{n}$ : 
Proposition 2.3. Take a standard parabolic subgroup $P=M \cdot N$ of $\operatorname{Sp}_{n}$ which is not equal to $B_{n}$. Let $\nu_{P}$ be the character of $Z_{M}(k) \backslash Z_{M}\left(\mathbb{A}_{k}\right)$ in Lemma 2.2. Then for every cusp form $f$ on $M\left(\mathbb{A}_{k}\right)$ with central character $\nu_{P}^{-1}, \varphi \in S\left(V\left(\mathbb{A}_{k}\right)^{n}\right)$, and $g \in \operatorname{Sp}_{n}\left(\mathbb{A}_{k}\right)$, we have

$$
\int_{Z_{M}\left(\mathbb{A}_{k}\right) M(k) \backslash M\left(\mathbb{A}_{k}\right)} I_{P}^{n}(m g, \varphi) f(m) d m=0 .
$$

Proof. Suppose $P$ is contained in $P_{r}, 0<r \leq n$, and $M \subset M_{r} \cong \mathrm{GL}_{r} \times \mathrm{Sp}_{n-r}$ is isomorphic to

$$
\mathrm{GL}_{r_{1}} \times \cdots \times \mathrm{GL}_{r_{\ell}} \times \mathrm{Sp}_{n-r}
$$

where $r_{1}+\cdots+r_{\ell}=r$. From the proof of Lemma 2.2, we have known that for every element $m=\left(a_{1}, \ldots, a_{\ell}, g^{\prime}\right) \in M\left(\mathbb{A}_{k}\right), g \in \operatorname{Sp}_{n}\left(\mathbb{A}_{k}\right)$, and $\varphi \in S\left(V\left(\mathbb{A}_{k}\right)^{n}\right)$,

$$
\begin{aligned}
I_{P}^{n}(m g, \varphi) & =I_{P_{r}}^{n}(m g, \varphi) \\
& =\left(\prod_{i=1}^{\ell} \chi_{V}\left(\operatorname{det} a_{i}\right)\left|\operatorname{det} a_{i}\right|_{\mathbb{A}_{k}}^{\frac{\operatorname{dim} V}{2}}\right) I^{n-r}\left(g^{\prime}, \widetilde{\varphi}\right) .
\end{aligned}
$$

Suppose $r_{i}>1$ for some $i$. Then $I_{P}^{n}$ is left invariant under $U_{i}\left(\mathbb{A}_{k}\right)$, where $U_{i}$ is the unipotent radical of a proper parabolic subgroup $P_{M}$ of $M$. Note that for every cusp form $f$ on $M\left(\mathbb{A}_{k}\right)$,

$$
\int_{U_{i}(k) \backslash U_{i}\left(\mathbb{A}_{k}\right)} f(u g) d u=0 .
$$

Therefore $\left(m \mapsto I_{P}^{n}(m g, \varphi)\right)$ is orthogonal to all cusp forms on $M\left(\mathbb{A}_{k}\right)$.

In the case when $r_{i}=1$ for all $1 \leq i \leq \ell$, we must have $r<n$ (as $\left.P \neq B_{n}\right)$. From Equation (2.1) we reduce to the case when $P=\mathrm{Sp}_{n}$. By Theorem A.5 in the Appendix A, we have that for every cusp form $f$ on $\operatorname{Sp}_{n}\left(\mathbb{A}_{k}\right)$,

$$
\int_{\mathrm{Sp}_{n}(k) \backslash \mathrm{Sp}_{n}\left(\mathbb{A}_{k}\right)} I^{n}(g, \varphi) \cdot f(g) d g=0 .
$$

This completes the proof.

\section{Siegel-EisEnStein SERIES}

Let $I_{\mathbb{A}_{k}}(s)$ be the space of smooth functions $\Phi$ on $\operatorname{Sp}_{n}\left(\mathbb{A}_{k}\right)$ satisfying that for elements $g \in \operatorname{Sp}_{n}\left(\mathbb{A}_{k}\right)$ and $\left(\begin{array}{cc}a & * \\ 0 & { }^{t} a^{-1}\end{array}\right) \in P_{n}\left(\mathbb{A}_{k}\right)$

$$
\Phi\left(\left(\begin{array}{cc}
a & * \\
0 & { }^{t} a^{-1}
\end{array}\right) g\right)=\chi_{V}(\operatorname{det} a)|\operatorname{det} a|_{\mathbb{A}_{k}}^{s+\frac{n+1}{2}} \cdot \Phi(g) .
$$

For $g \in \operatorname{Sp}_{n}\left(\mathbb{A}_{k}\right)$ and $\Phi \in I_{\mathbb{A}_{k}}(s)$, it is known that the Eisenstein series

$$
E(g, s, \Phi):=\sum_{\gamma \in P_{n}(k) \backslash \operatorname{Sp}_{n}(k)} \Phi(\gamma g)
$$

converges absolutely for $\operatorname{Re}(s)>(n+1) / 2$. From the Iwasawa decomposition

$$
\operatorname{Sp}_{n}\left(\mathbb{A}_{k}\right)=P_{n}\left(\mathbb{A}_{k}\right) \cdot \operatorname{Sp}_{n}\left(O_{\mathbb{A}_{k}}\right),
$$

we can extend $\Phi$ to a standard section (which is still denoted by $\Phi$ ), i.e. for all $s^{\prime} \in \mathbb{C}$, $a \in \mathrm{GL}_{n}\left(\mathbb{A}_{k}\right), \kappa \in \operatorname{Sp}_{n}\left(O_{\mathbb{A}_{k}}\right)$,

$$
\Phi\left(\left(\begin{array}{cc}
a & * \\
0 & { }^{t} a^{-1}
\end{array}\right) \kappa, s^{\prime}\right)=\chi_{V}(\operatorname{det} a)|\operatorname{det} a|_{\mathbb{A}_{k}}^{s^{\prime}+\frac{n+1}{2}} \cdot \Phi(\kappa) .
$$

It is known that for $g \in \operatorname{Sp}_{n}\left(\mathbb{A}_{k}\right), E(g, s, \Phi)$ can be extended to a meromorphic function of $s \in \mathbb{C}$ (in fact, a rational function in $q^{-s}$ ).

In this section, our aim is to show that $E(g, s, \Phi)$ is also concentrated on the Borel subgroup $B_{n}$ for every section $\Phi \in I_{\mathbb{A}_{k}}(s)$ (in Proposition 3.2). 
3.1. Constant terms of Eisenstein series. Let $P$ be a standard parabolic subgroup of $\mathrm{Sp}_{n}$, i.e. $P$ is a parabolic subgroup of $\mathrm{Sp}_{n}$ containing $B_{n}$. Then $P=M \cdot N$, where $M$ is the Levi subgroup of $P$ and $N$ is the unipotent radical of $P$. For $g \in \operatorname{Sp}_{n}\left(\mathbb{A}_{k}\right)$ and $\Phi \in I_{\mathbb{A}_{k}}(s)$, the constant term of $E$ along $P$ is defined by

$$
E_{P}(g, s, \Phi):=\int_{N(k) \backslash N\left(\mathbb{A}_{k}\right)} E_{P}(n g, s, \Phi) d n .
$$

Here the measure $d n$ is induced from the Haar measure of $N\left(\mathbb{A}_{k}\right)$ normalized so that the volume of $N(k) \backslash N\left(\mathbb{A}_{k}\right)$ is 1 . Let $W_{M}$ be the Weyl group of $M$ with respect to $T_{n}$, and denote by $\Delta_{M}^{+}$the set of positive roots with respect to the Borel subgroup $B_{n} \cap M$ of $M$. Then it is known that

$$
W_{M_{n}, M}:=\left\{w \in W_{\mathrm{Sp}_{n}} \mid w^{-1} \alpha \in \Delta_{n}^{+} \text {for all } \alpha \in \Delta_{M_{n}}^{+} \text {, and } w \alpha \in \Delta_{n}^{+} \text {for all } \alpha \in \Delta_{M}^{+}\right\}
$$

forms a set of double coset representatives of $W_{M_{n}} \backslash W_{\mathrm{Sp}_{n}} / W_{M}$.

Lemma 3.1. For each $w \in W_{M_{n}, M}$, let $M_{w}^{\prime \prime}:=w^{-1} P_{n} w \cap M$ and $N_{w}^{\prime \prime}:=w^{-1} P_{n} w \cap N$. Then

$$
E_{P}(g, s, \Phi)=\sum_{w \in W_{M_{n}, M}}\left(\sum_{\gamma^{\prime} \in M_{w}^{\prime \prime}(k) \backslash M(k)} \Phi_{w}\left(\gamma^{\prime} g\right)\right),
$$

where

$$
\Phi_{w}(g):=\int_{N_{w}^{\prime \prime}\left(\mathbb{A}_{k}\right) \backslash N\left(\mathbb{A}_{k}\right)} \Phi(w n g) d n .
$$

Proof. Note that $W_{M_{n}, M}$ also forms a set of double coset representatives of $P_{n}(k) \backslash \operatorname{Sp}_{n}(k) / P(k)$. Moreover, for each $w \in W_{M_{n}, M}$ we have the following bijection

$$
\left(N_{w}^{\prime \prime}(k) \backslash N(k)\right) \times\left(M_{w}^{\prime \prime}(k) \backslash M(k)\right) \cong w^{-1} P(k) w \cap P(k) \backslash P(k) .
$$

Therefore

$$
\begin{aligned}
E_{P}(g, s, \Phi) & =\int_{N(k) \backslash N\left(\mathbb{A}_{k}\right)} \sum_{w \in P_{n}(k) \backslash \mathrm{Sp}_{n}(k) / P(k)} \sum_{\gamma_{w} \in w^{-1} P_{n}(k) w \cap P(k) \backslash P(k)} \Phi\left(w \gamma_{w} n g\right) d n \\
& =\sum_{w \in W_{M_{n}, M}}\left(\sum_{\gamma^{\prime} \in M_{w}^{\prime \prime}(k) \backslash M(k)} \int_{N(k) \backslash N\left(\mathbb{A}_{k}\right)} \sum_{n^{\prime} \in N_{w}^{\prime \prime}(k) \backslash N(k)} \Phi\left(w n^{\prime} \gamma^{\prime} n g\right) d n\right) .
\end{aligned}
$$

It is observed that

$$
\begin{aligned}
& \int_{N(k) \backslash N\left(\mathbb{A}_{k}\right)} \sum_{n^{\prime} \in N_{w}^{\prime \prime}(k) \backslash N(k)} \Phi\left(w n^{\prime} \gamma^{\prime} n g\right) d n \\
= & \int_{N(k) \backslash N\left(\mathbb{A}_{k}\right)} \sum_{n^{\prime} \in N_{w}^{\prime \prime}(k) \backslash N(k)} \Phi\left(w\left(n^{\prime} n\right) \gamma^{\prime} g\right) d n \\
= & \int_{N_{w}^{\prime \prime}(k) \backslash N\left(\mathbb{A}_{k}\right)} \Phi\left(w n \gamma^{\prime} g\right) d n \\
= & \Phi_{w}\left(\gamma^{\prime} g\right) .
\end{aligned}
$$

The last equality is from

$$
\Phi\left(w n^{\prime \prime} g\right)=\Phi(w g), \quad \forall n^{\prime \prime} \in N_{w}^{\prime \prime}\left(\mathbb{A}_{k}\right) .
$$

For $w \in W_{M_{n}, M}$, there exists a character $\mu_{w}$ on $M_{w}^{\prime \prime}\left(\mathbb{A}_{k}\right)$ trivial on $M_{w}^{\prime \prime}(k)$ such that for $m \in M_{w}^{\prime \prime}\left(\mathbb{A}_{k}\right)$

$$
\Phi_{w}(m g)=\mu_{w}(m) \Phi_{w}(g) .
$$


Note that $M_{w}^{\prime \prime}$ is a standard parabolic subgroup of $M$ (with respect to $B_{n} \cap M$ ) for any $w \in W_{M_{n}, M}$. Let $\nu_{w}$ be the restriction of $\mu_{w}$ on the center $Z_{M}\left(\mathbb{A}_{k}\right)$ of $M\left(\mathbb{A}_{k}\right)$. Then we can write

$$
E_{P}(g, s, \Phi)=\sum_{\text {character } \nu \text { on } Z_{M}\left(\mathbb{A}_{k}\right)} E_{P, \nu}(g, s, \Phi),
$$

where

$$
E_{P, \nu}(g, s, \Phi):=\sum_{w \in W_{M_{n}, M}, \nu_{w}=\nu}\left(\sum_{\gamma \in M_{w}^{\prime \prime}(k) \backslash M(k)} \Phi_{w}(\gamma g)\right) .
$$

Since $\mu_{w}$ is trivial on $U_{M_{w}^{\prime \prime}}\left(\mathbb{A}_{k}\right)$ where $U_{M_{w}^{\prime \prime}}$ is the unipotent radical of $M_{w}^{\prime \prime}$ when $M_{w}^{\prime \prime} \neq M$ and we take $U_{M_{w}^{\prime \prime}}=B_{n} \cap M$ if $M_{w}^{\prime \prime}=M$, we have the following result.

Proposition 3.2. Suppose a function $\Phi \in I_{\mathbb{A}_{k}}(s)$ and $g \in \operatorname{Sp}_{n}\left(\mathbb{A}_{k}\right)$ are given. Let $P \supsetneq B_{n}$ be a standard parabolic subgroup of $\mathrm{Sp}_{n}$. Then for any cusp form $f$ on $M\left(\mathbb{A}_{k}\right)$ (where $M$ is the Levi subgroup of $P$ ) with central character $\nu^{-1}$, we have

$$
\int_{Z_{M}\left(\mathbb{A}_{k}\right) M(k) \backslash M\left(\mathbb{A}_{k}\right)} E_{P, \nu}(m g, s, \Phi) f(m) d m=0 .
$$

Corollary 3.3. For every section $\Phi$ and every standard parabolic subgroup $P$ of $\mathrm{Sp}_{n}, E(g, s, \Phi)$ and $E_{P}(g, s, \Phi)$ have the same set of poles. More precisely, for $s_{0} \in \mathbb{C}$, let

$$
\operatorname{ord}_{s=s_{0}} E(\cdot, s, \Phi)=\min _{g \in \operatorname{Sp}_{n}\left(\mathbb{A}_{k}\right)}\left\{\operatorname{ord}_{s=s_{0}} E(g, s, \Phi)\right\}
$$

and

$$
\operatorname{ord}_{s=s_{0}} E_{P}(\cdot, s, \Phi)=\min _{g \in \operatorname{Sp}_{n}\left(\mathbb{A}_{k}\right)}\left\{\operatorname{ord}_{s=s_{0}} E_{P}(g, s, \Phi)\right\}
$$

Then

$$
\operatorname{ord}_{s=s_{0}} E(\cdot, s, \Phi)=\operatorname{ord}_{s=s_{0}} E_{P}(\cdot, s, \Phi), \quad \forall s_{0} \in \mathbb{C} .
$$

Proof. From the definition of $E_{P}(g, s, \Phi)$, it is clear that

$$
\operatorname{ord}_{s=s_{0}} E_{P}(\cdot, s, \Phi) \geq \operatorname{ord}_{s=s_{0}} E(\cdot, s, \Phi) .
$$

Write $P=M \cdot N$, where $N$ is the unipotent radical of $P$ and $M$ is the Levi subgroup. We also have

$$
\operatorname{ord}_{s=s_{0}} E_{B_{n}}(\cdot, s, \Phi) \geq \operatorname{ord}_{s=s_{0}} E_{P}(\cdot, s, \Phi),
$$

as for every $g \in \operatorname{Sp}_{n}\left(\mathbb{A}_{k}\right)$

$$
E_{B_{n}}(g, s, \Phi)=\int_{B_{n}(k) N\left(\mathbb{A}_{k}\right) \backslash B_{n}\left(\mathbb{A}_{k}\right)} E_{P}(n g, s, \Phi) d n .
$$

It suffices to show that

$$
\operatorname{ord}_{s=s_{0}} E(\cdot, s, \Phi) \geq \operatorname{ord}_{s=s_{0}} E_{B_{n}}(\cdot, s, \Phi) .
$$

Let $\ell=\operatorname{ord}_{s=s_{0}} E(\cdot, s, \Phi)$ and $\ell^{\prime}=\operatorname{ord}_{s=s_{0}} E_{B_{n}}(\cdot, s, \Phi)$. If $\ell<\ell^{\prime}$, then the function $f(g):=\lim _{s \rightarrow s_{0}}\left(s-s_{0}\right)^{-\ell} E(g, s, \Phi)$ would have

$$
f_{B_{n}}(g)=\int_{U_{n}(k) \backslash U_{n}\left(\mathbb{A}_{k}\right)} f(u g) d u=0 .
$$

By Proposition 3.2, $f$ is also concentrated on $B_{n}$. Therefore $f \equiv 0$ (as $f$ is a cusp form on $\mathrm{Sp}_{n}\left(\mathbb{A}_{k}\right)$ which is also orthogonal to all cusp forms). Therefore the proof is complete. 
Take a Schwartz function $\varphi \in S\left(V\left(\mathbb{A}_{k}\right)^{n}\right)$. For any $g=\left(\begin{array}{cc}a & * \\ 0 & { }^{t} a^{-1}\end{array}\right) \cdot \kappa \in \operatorname{Sp}_{n}\left(\mathbb{A}_{k}\right)$ where $a \in \mathrm{GL}_{n}\left(\mathbb{A}_{k}\right)$ and $\kappa \in \operatorname{Sp}_{n}\left(O_{\mathbb{A}_{k}}\right)$, set $s_{(n)}:=\operatorname{dim}(V) / 2-(n+1) / 2$ and

$$
\Phi_{\varphi}(g, s):=|\operatorname{det} a|_{\mathbb{A}_{k}}^{s-s_{(n)}} \cdot(\omega(g) \varphi)(0) .
$$

Then $\Phi_{\varphi}$ is in $I_{\mathbb{A}_{k}}(s)$. We call $\Phi_{\varphi}$ the Siegel section associated to $\varphi$ and $E\left(g, s, \Phi_{\varphi}\right)$ the SiegelEisenstein series associated to $\varphi$. To show that $E\left(g, s, \Phi_{\varphi}\right)$ is holomorphic at $s=s_{(n)}$ for every Siegel section $\Phi_{\varphi}$, by Corollary 3.3 it suffices to get

$$
\operatorname{ord}_{s=s_{(n)}} E_{P_{n}}\left(\cdot, s, \Phi_{\varphi}\right) \geq 0 .
$$

In the next two sections, we study the analytic behavior of $E_{P_{n}}\left(g, s, \Phi_{\varphi}\right)$ at $s=s_{(n)}$, and prove its holomorphic property.

4. The analytic Behavior of $E_{P_{n}}\left(g, s, \Phi_{\varphi}\right)$ I: Intertwining operators

For $0 \leq r \leq n$, let

$$
w_{r}:=\left(\begin{array}{cccc}
I_{n-r} & 0 & 0 & 0 \\
0 & 0 & 0 & I_{r} \\
0 & 0 & I_{n-r} & 0 \\
0 & -I_{r} & 0 & 0
\end{array}\right) \in \operatorname{Sp}_{n} .
$$

Then $\left\{w_{0}, \ldots, w_{n}\right\}$ is a set of double coset representatives of $W_{M_{n}} \backslash W_{\mathrm{Sp}_{n}} / W_{M_{n}}$, and we have $\mathrm{Sp}_{n}=\coprod_{r=0}^{n} P_{n} w_{r} P_{n}$. Moreover, $M_{w_{r}}^{\prime \prime}=w_{r}^{-1} P_{n} w_{r} \cap M_{n}=\left\{\mathfrak{m}(a): a \in Q_{r}\right\}$ where

$$
Q_{r}:=\left\{\left(\begin{array}{ll}
a & * \\
0 & d
\end{array}\right) \in \mathrm{GL}_{n} \mid a \in \mathrm{GL}_{n-r}, d \in \mathrm{GL}_{r}\right\},
$$

and

$$
\begin{aligned}
& N_{w_{r}}^{\prime \prime}=w_{r}^{-1} P_{n} w_{r} \cap N_{n}=\left\{\mathfrak{n}\left(\begin{array}{cc}
y & z \\
t z & 0
\end{array}\right) \mid y \in \operatorname{Sym}_{n-r}, z \in \operatorname{Mat}_{(n-r) \times r}\right\}, \\
& N_{w_{r}}^{\prime \prime} \backslash N_{n} \cong N_{r}^{\prime}:=\left\{\mathfrak{n}\left(\begin{array}{ll}
0 & 0 \\
0 & b
\end{array}\right) \mid b \in \operatorname{Sym}_{r}\right\} .
\end{aligned}
$$

Here $\mathfrak{m}$ and $\mathfrak{n}$ are the isomorphisms introduced in Section 1.2.

Take a section $\Phi \in I_{\mathbb{A}_{k}}(s)$. By Lemma 3.1, we can write $E_{P_{n}}(g, s, \Phi)$ as

$$
\sum_{r=0}^{n} E_{P_{n}}^{(r)}(g, s, \Phi)
$$

where

$$
E_{P_{n}}^{(r)}(g, s, \Phi):=\sum_{\gamma \in Q_{r}(k) \backslash \mathrm{GL}_{n}(k)} \Phi^{(r)}(\mathfrak{m}(\gamma) g, s)
$$

with

Notice that

$$
\Phi^{(r)}(g, s):=\int_{N_{r}^{\prime}\left(\mathbb{A}_{k}\right)} \Phi\left(w_{r} n g, s\right) d n .
$$

$$
E_{P_{n}}^{(0)}(g, s, \Phi)=\Phi(g, s), \quad E_{P_{n}}^{(n)}(g, s, \Phi)=(M(s) \Phi)(g, s)
$$

where $M(s): I_{\mathbb{A}_{k}}(s) \rightarrow I_{\mathbb{A}_{k}}(-s)$ is the intertwining operator defined by

and

$$
(M(s) \Phi)(g, s):=\int_{N_{n}\left(\mathbb{A}_{k}\right)} \Phi\left(w_{n} n g, s\right) d n,
$$

$$
a \mapsto E_{P_{n}}^{(r)}(\mathfrak{m}(a) g, s, \Phi), \quad \forall a \in \mathrm{GL}_{n}\left(\mathbb{A}_{k}\right)
$$

is an Eisenstein series on $\mathrm{GL}_{n}\left(\mathbb{A}_{k}\right)$ for $0<r<n$. 
In this section, we review the meromorphic continuation of the intertwining operator $M(s)$, and show that $E_{P_{n}}^{(n)}(g, s, \Phi)$ is holomorphic at $s=s_{(n)}$ when $\Phi=\Phi_{\varphi}$ is a Siegel section.

4.1. The intertwining operator $M(s)$. For each place $v$ of $k$, let $I_{v}(s)$ be the space of smooth functions $\Phi_{v}$ on $\operatorname{Sp}_{n}\left(k_{v}\right)$ satisfying that for $g \in \operatorname{Sp}_{n}\left(k_{v}\right)$ and $\left(\begin{array}{cc}a & * \\ 0 & { }^{t} a^{-1}\end{array}\right) \in P_{n}\left(k_{v}\right)$,

$$
\Phi_{v}\left(\left(\begin{array}{cc}
a & * \\
0 & { }^{t} a^{-1}
\end{array}\right) g\right)=\chi_{V, v}(\operatorname{det} a)|\operatorname{det} a|_{v}^{s+\frac{n+1}{2}} \Phi_{v}(g) .
$$

Given $\Phi_{v} \in I_{v}(s)$, we can extend $\Phi_{v}$ to be a standard section, i.e. for $s^{\prime} \in \mathbb{C}$,

$$
\Phi_{v}\left(\left(\begin{array}{cc}
a & * \\
0 & { }^{t} a^{-1}
\end{array}\right) \kappa_{v}\right):=\chi_{V, v}(\operatorname{det} a)|\operatorname{det} a|_{v}^{s^{\prime}+\frac{n+1}{2}} \Phi_{v}\left(\kappa_{v}\right), \quad \forall a \in \mathrm{GL}_{n}\left(k_{v}\right), \kappa_{v} \in \operatorname{Sp}_{n}\left(O_{v}\right) .
$$

Define the intertwining operator $M_{v}(s): I_{v}(s) \rightarrow I_{v}(-s)$ by

$$
M_{v}(s) \Phi_{v}(g):=\int_{N_{n}\left(k_{v}\right)} \Phi_{v}\left(w_{n} n_{v} g\right) d n_{v}
$$

which converges when $s>(n-1) / 2$. We state the known facts we need in the following:

Lemma 4.1. (cf. [5]) Let

$$
a_{n, v}(s):=L_{v}\left(s+\frac{n+1}{2}-n, \chi_{V, v}\right) \prod_{i=1}^{\lfloor n / 2\rfloor} \zeta_{k, v}(2 s-n+2 i)
$$

and

$$
b_{n, v}(s)=L_{v}\left(s+\frac{n+1}{2}, \chi_{V, v}\right) \prod_{i=1}^{\lfloor n / 2\rfloor} \zeta_{k, v}(2 s+n-2 i+1)
$$

where

$$
\zeta_{k, v}(s):=\left(1-q_{v}^{-s}\right)^{-1} \quad \text { and } \quad L_{v}\left(s, \chi_{V, v}\right):=\left(1-\alpha_{v}\left(\chi_{V, v}\right) q_{v}^{-s}\right)^{-1}
$$

with

$$
\alpha_{v}\left(\chi_{V, v}\right):= \begin{cases}\chi_{V, v}\left(\pi_{v}\right), & \text { if } \chi_{V, v} \text { is unramified, } \\ 0, & \text { otherwise. }\end{cases}
$$

(i) For any standard section $\Phi_{v} \in I_{v}(s)$ and $g \in \operatorname{Sp}_{n}\left(k_{v}\right)$,

$$
\frac{1}{a_{n, v}(s)} M_{v}(s) \Phi_{v}(g, s)
$$

can be extended to an entire function of $s$.

(ii) Suppose $\chi_{V, v}$ is unramified. Let $\Phi_{v}^{0}(g, s) \in I_{v}(s)$ be the standard section such that

$$
\Phi_{v}^{0}\left(\kappa_{v}, s\right)=1, \quad \forall \kappa_{v} \in \operatorname{Sp}_{n}\left(O_{v}\right) .
$$

Then we have

$$
M_{v}(s) \Phi_{v}^{0}(g, s)=\operatorname{vol}\left(N_{n}\left(O_{v}\right), d n_{v}\right) \cdot \frac{a_{n, v}(s)}{b_{n, v}(s)} \cdot \Phi_{v}^{0}(g,-s), \quad \forall g \in \operatorname{Sp}_{n}\left(k_{v}\right) .
$$

Recall that $I_{\mathbb{A}_{k}}(s)$ is the restricted tensor product of $I_{v}(s)$ with respect to $\left\{\Phi_{v}^{0}\right\}$. We normalize the Haar measure $d n_{v}$ on $N_{n}\left(k_{v}\right)$ such that $\operatorname{vol}\left(N_{n}\left(O_{v}\right)\right)=1$ for almosrt all places $v$ and the Haar measure $d n=\prod_{v} d n_{v}$ on $N_{n}\left(\mathbb{A}_{k}\right)$ satisfies $\operatorname{vol}\left(N_{n}(k) \backslash N_{n}\left(\mathbb{A}_{k}\right), d n\right)=1$. Then $M(s)=\otimes M_{v}(s)$, which converges absolutely on $\operatorname{Re}(s)>(n+1) / 2$. In particular, for a factorizable section $\Phi=\otimes_{v} \Phi_{v} \in I_{\mathbb{A}_{k}}(s)$, let $\Sigma(\Phi, d n)$ be the finite set of places $v$ of $k$ such that $\Phi_{v} \neq \Phi_{v}^{0}$ or $\operatorname{vol}\left(N_{n}\left(O_{v}\right), d n_{v}\right) \neq 1$. Then by Lemma 4.1 (ii), $M(s) \Phi(g, s)$ can be expressed by

$$
\frac{a_{n}(s)}{b_{n}(s)} \cdot\left[\left(\otimes_{v \notin \Sigma(\Phi, d n)} \Phi_{v}^{0}\left(g_{v},-s\right)\right) \otimes\left(\otimes_{v \in \Sigma(\Phi, d n)} \frac{b_{n, v}(s)}{a_{n, v}(s)} M_{v}(s) \Phi_{v}\left(g_{v}, s\right)\right)\right],
$$


where

$$
a_{n}(s):=\prod_{v} a_{n, v}(s) \quad \text { and } \quad b_{n}(s):=\prod_{v} b_{n, v}(s) .
$$

By Lemma 4.1 (i), we have the meromorphic continuation of $M(s) \Phi(g, s)$ for each element $g \in \operatorname{Sp}_{n}\left(\mathbb{A}_{k}\right)$.

\section{Lemma 4.2 .}

(i) Suppose $\chi_{V}$ is non-trivial. Then when $\operatorname{dim}(V)=2$ we have

$$
\operatorname{ord}_{s=s_{(n)}} \frac{a_{n}(s)}{b_{n}(s)}= \begin{cases}0, & \text { if } n=1, \\ +1, & \text { if } n>1 ;\end{cases}
$$

when $\operatorname{dim}(V)=4$,

$$
\operatorname{ord}_{s=s_{(n)}} \frac{a_{n}(s)}{b_{n}(s)}= \begin{cases}0, & \text { if } n=1 \text { or } 3, \\ -1, & \text { if } n=2, \\ 1, & \text { if } n>3 .\end{cases}
$$

(ii) When $\chi_{V}$ is trivial, we have $\operatorname{dim}(V)=4$ and

$$
\operatorname{ord}_{s=s_{(n)}} \frac{a_{n}(s)}{b_{n}(s)}= \begin{cases}-1, & \text { if } n=1, \\ -2, & \text { if } n=2, \\ 0, & \text { if } n=3, \\ +1, & \text { if } n>3 .\end{cases}
$$

Recall that $S\left(V\left(\mathbb{A}_{k}\right)^{n}\right)$ can be viewed as the restricted tensor product $\otimes_{v}^{\prime} S\left(V\left(k_{v}\right)^{n}\right)$ with respect to $\left\{\varphi_{v}^{0}\right\}_{v}$, where the functions $\varphi_{v}^{0}$ are chosen in Section 1.3. Suppose a factorizable Schwartz function $\varphi=\otimes_{v} \varphi_{v} \in S\left(V\left(\mathbb{A}_{k}\right)^{n}\right)$ is given. Then the associated section $\Phi_{\varphi}=\otimes_{v} \Phi_{\varphi_{v}}$ is also factorizable.

Proposition 4.3. For each place $v$ of $k$,

$$
\frac{b_{n, v}(s)}{a_{n, v}(s)} M_{v}(s) \Phi_{\varphi_{v}}\left(g_{v}, s\right)
$$

is holomorphic at $s=s_{(n)}$ for all $g \in \operatorname{Sp}_{n}\left(k_{v}\right)$ and $\varphi_{v} \in S\left(V\left(k_{v}\right)^{n}\right)$.

Remark. The above proposition does not hold for all sections of $I_{v}(s)$ in general, as

$$
\operatorname{ord}_{s=s_{(n)}} b_{n, v}(s)= \begin{cases}-1, & \text { if } \operatorname{dim}(V)<n+1, \\ 0, & \text { if } \operatorname{dim}(V) \geq n+1 .\end{cases}
$$

Proof. We define an intertwining operator $T_{v}$ from $S\left(V\left(k_{v}\right)^{n}\right)$ to $I_{v}\left(-s_{(n)}\right)$ (as representations of $\left.\operatorname{Sp}_{n}\left(k_{v}\right)\right)$ by

$$
\varphi_{v} \longmapsto \frac{1}{a_{n, v}\left(s_{(n)}\right)} M_{v}\left(s_{(n)}\right) \Phi_{\varphi_{v}} .
$$

When $\operatorname{dim}(V)<n+1$, this intertwining operator $T_{v}$ must be zero by the following lemma (Lemma 4.4), which tells us that $a_{n, v}(s)^{-1} M_{n, n, v}(s) \Phi_{\varphi_{v}}(g, s)$ has a zero at $s=s_{(n)}$ for all $\varphi \in S\left(V\left(k_{v}\right)^{n}\right)$ and $g \in \operatorname{Sp}_{n}\left(k_{v}\right)$. Therefore the result holds.

\section{Lemma 4.4. Let}

$$
\ell(n, v):=\operatorname{dim}_{\mathbb{C}} \operatorname{Hom}_{\mathrm{Sp}_{n}\left(k_{v}\right) \times \mathrm{O}(V)\left(k_{v}\right)}\left(S\left(V\left(k_{v}\right)^{n}\right), I_{v}\left(-s_{(n)}\right) \otimes \mathbf{1}\right),
$$

where 1 is the trivial representation of $\mathrm{O}(V)\left(k_{v}\right)$. Then

(i) When $\operatorname{dim}(V)<n+1$, we have $\ell(n, v)=0$.

(ii) When $\operatorname{dim}(V)=2$ and $n=1, \ell(n, v) \leq 1$. 
(iii) When $\operatorname{dim}(V)=4$ and $n=1$ or 2 ,

$$
\ell(n, v) \leq \begin{cases}n, & \text { if } V\left(k_{v}\right) \text { is isotropic, } \\ 0, & \text { if } V\left(k_{v}\right) \text { is anisotropic. }\end{cases}
$$

(iv) When $\operatorname{dim}(V)=4$ and $n=3$, we have $\ell(n, v) \leq 1$.

Proof. This is a consequence of Proposition B.1. The proof is given in Appendix B (cf. Corollary B.3 and Remark B.4).

The above lemma implies immediately that:

Corollary 4.5. If $\operatorname{dim}(V) \geq n+1$ and $\ell(n, v)=0$, then the meromorphic function

$$
\frac{b_{n, v}(s)}{a_{n, v}(s)} M_{n, n, v}(s) \Phi_{\varphi}(g, s)
$$

has a zero at $s=s_{(n)}$ for all $g \in \operatorname{Sp}_{n}\left(k_{v}\right)$ and $\varphi \in S\left(V\left(k_{v}\right)^{n}\right)$.

Recall that given $\varphi=\otimes_{v} \varphi_{v} \in S\left(V\left(\mathbb{A}_{k}\right)^{n}\right), M(s) \Phi_{\varphi}(g, s)$ can be expressed by

$$
\frac{a_{n}(s)}{b_{n}(s)} \cdot\left[\left(\otimes_{v \notin \Sigma\left(\Phi_{\varphi}, d n\right)} \Phi_{\varphi_{v}}^{0}\left(g_{v},-s\right)\right) \otimes\left(\otimes_{v \in \Sigma\left(\Phi_{\varphi_{v}}, d n\right)} \frac{b_{n, v}(s)}{a_{n, v}(s)} M_{v}(s) \Phi_{\varphi_{v}}\left(g_{v}, s\right)\right)\right] .
$$

Note that when $\operatorname{dim}(V)=4$ and $\chi_{V}$ is non-trivial (resp. other cases), there exists at least one (resp. two) places of $k$ such that $\left(V, Q_{V}\right)$ is anisotropic over $k_{v}$. Since any Schwartz function $\varphi \in S\left(V\left(\mathbb{A}_{k}\right)^{n}\right)$ is a linear combination of factorizable functions, we finally arrive at:

Proposition 4.6. For each Siegel section $\Phi_{\varphi} \in I_{\mathbb{A}_{k}}(s), M(s) \Phi_{\varphi}(g, s)$ is holomorphic at $s=s_{(n)}$ for all $g \in \operatorname{Sp}_{n}\left(\mathbb{A}_{k}\right)$. Moreover,

$$
M\left(s_{(n)}\right) \Phi_{\varphi}\left(g, s_{(n)}\right)=0 \quad \text { for all } g \in \operatorname{Sp}_{n}\left(\mathbb{A}_{k}\right)
$$

except for the following cases:

(i) when $\operatorname{dim}(V)=2$ and $n=1$;

(ii) when $\operatorname{dim}(V)=4$ and $n=2$ or 3 .

4.2. The intertwining operator $M_{n, r}(s)$. Fix an integer $r$ with $0<r<n$. We consider the standard parabolic subgroup $P_{n}^{(r)}:=M_{n}^{(r)} N_{n}^{(r)}$ contained in $P_{n}$, where the Levi subgroup $M_{n}^{(r)} \subset M_{n}$ is equal to

$$
\left\{\mathfrak{m}\left(\begin{array}{cc}
a_{1} & 0 \\
0 & a_{2}
\end{array}\right) \mid a_{1} \in \mathrm{GL}_{n-r}, a_{2} \in \mathrm{GL}_{r}\right\} .
$$

For each pair of Hecke characters $\mu_{1}$ and $\mu_{2}$ on $k^{\times} \backslash \mathbb{A}_{k}^{\times}$, Let $I_{n, r}\left(\mu_{1}, \mu_{2}\right)$ be the space of smooth functions $f$ on $\operatorname{Sp}_{n}\left(\mathbb{A}_{k}\right)$ satisfying that for $\left(a_{1}, a_{2}\right) \in \mathrm{GL}_{n-r} \times \mathrm{GL}_{r}$ and $n^{\prime} \in N_{n}^{(r)}$,

$$
f\left(\mathfrak{m}\left(\begin{array}{cc}
a_{1} & 0 \\
0 & a_{2}
\end{array}\right) n^{\prime} g\right)=\mu_{1}\left(a_{1}\right) \mu_{2}\left(a_{2}\right) \frac{\left|\operatorname{det} a_{1}\right|_{\mathbb{A}_{k}}^{\frac{n+r+1}{2}}}{\left|\operatorname{det} a_{2}\right|_{\mathbb{A}_{k}}^{\frac{r+1}{2}}} f(g) .
$$

For a section $\Phi \in I_{\mathbb{A}_{k}}(s)$, define

$$
M_{n, r}(s) \Phi(g, s):=\int_{N_{r}^{\prime}\left(\mathbb{A}_{k}\right)} \Phi\left(w_{r} n g, s\right) d n,
$$

where the Haar measure $d n$ is normalized so that the volume of $N_{r}^{\prime}(k) \backslash N_{r}^{\prime}\left(\mathbb{A}_{k}\right)$ is 1 . When $\operatorname{Re}(s)>(n+1) / 2$, it is clear that $M_{n, r}(s) \Phi(\cdot, s)$ is a section in $I_{n, r}(s)$, where

$$
I_{n, r}(s):=I_{n, r}\left(|\cdot|_{\mathbb{A}_{k}}^{s-\frac{r}{2}},|\cdot|_{\mathbb{A}_{k}}^{-s+\frac{n-r}{2}}\right) .
$$

We also set $I_{n, 0}(s):=I_{\mathbb{A}_{k}}(s)$ and $I_{n, n}(s):=I_{\mathbb{A}_{k}}(-s)$, and $M_{n, n}(s):=M(s)$ introduced in Section 4.1. 
For any $\kappa \in \operatorname{Sp}_{n}\left(O_{\mathbb{A}_{k}}\right)$, let $\rho(\kappa)$ denote the left action of $\kappa$ by right multiplication on $I_{\mathbb{A}_{k}}(s)$ and $I_{n, r}(s)$. This action is independent of $s$. Let $\Phi \in I_{\mathbb{A}_{k}}(s)$ be a standard section. Given $\kappa \in \operatorname{Sp}_{n}\left(O_{\mathbb{A}_{k}}\right)$, let $\Phi^{\prime}:=\rho(\kappa) \Phi$, which is also a standard section. Define the inclusion $i: \mathrm{Sp}_{r} \hookrightarrow \mathrm{Sp}_{n}$ by

$$
\left(\begin{array}{ll}
a & b \\
c & d
\end{array}\right) \longmapsto\left(\begin{array}{cccc}
I_{n-r} & & & \\
& a & & b \\
& & I_{n-r} & \\
& c & & d
\end{array}\right) .
$$

Then we have the following commutative diagram:

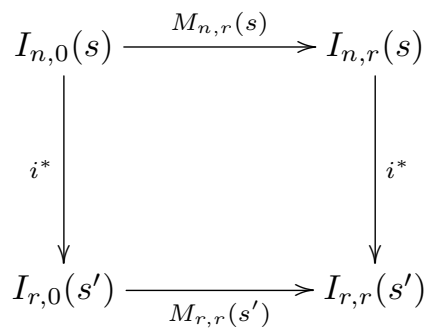

where $s^{\prime}=s+\frac{n-r}{2}$. For each $g \in \operatorname{Sp}_{n}\left(\mathbb{A}_{k}\right)$, write $g=n(b) m(a) \kappa$ where $n(b) \in N_{n}\left(\mathbb{A}_{k}\right)$, $a=\left(\begin{array}{cc}a_{1} & * \\ 0 & a_{2}\end{array}\right) \in Q_{r}\left(\mathbb{A}_{k}\right)$, and $\kappa \in \operatorname{Sp}_{n}\left(O_{\mathbb{A}_{k}}\right)$. Then

$$
\begin{aligned}
M_{n, r}(s) \Phi(g, s) & =\left|\operatorname{det} a_{1}\right|_{\mathbb{A}_{k}}^{s+\frac{n+1}{2}} \cdot\left|\operatorname{det} a_{2}\right|_{\mathbb{A}_{k}}^{-s+\frac{n+1}{2}} M_{n, r}(s)(\rho(\kappa) \Phi)(1, s) \\
& =\left|\operatorname{det} a_{1}\right|_{\mathbb{A}_{k}}^{s+\frac{n+1}{2}} \cdot\left|\operatorname{det} a_{2}\right|_{\mathbb{A}_{k}}^{-s+\frac{n+1}{2}} M_{r, r}\left(s^{\prime}\right)\left(i^{*}(\rho(\kappa) \Phi)\right)\left(1, s^{\prime}\right) .
\end{aligned}
$$

This gives the meromorphic continuation of $M_{n, r}(s) \Phi$ for each standard section $\Phi$, and

$$
\operatorname{ord}_{s=s_{(n)}} M_{n, r}(s) \Phi(g, s)=\operatorname{ord}_{s^{\prime}=s_{(r)}} M_{r, r}\left(s^{\prime}\right)\left(i^{*}(\rho(\kappa) \Phi)\right)\left(1, s^{\prime}\right) .
$$

In particular, suppose $\Phi$ is factorizable. Write $\Phi=\otimes_{v} \Phi_{v}$. Recall that $\Sigma(\Phi, d n)$ is the finite set of places of $k$ such that $\Phi_{v}=\Phi_{v}^{0}$ and $\operatorname{vol}\left(N_{n}\left(O_{v}\right)\right)=1$ when $v \notin \Sigma(\Phi, d n)$. Then $M_{n, r}(s) \Phi$ is equal to

$$
\frac{a_{r}\left(s^{\prime}\right)}{b_{r}\left(s^{\prime}\right)} \cdot\left[\left(\otimes_{v \notin \Sigma(\Phi, d n)} \Phi_{v}^{0}\right) \otimes\left(\otimes_{v \in \Sigma(\Phi, d n)} \frac{b_{r, v}\left(s^{\prime}\right)}{a_{r, v}\left(s^{\prime}\right)} M_{n, r, v}(s) \Phi_{v}\right)\right]
$$

5. The analytic Behavior of $E_{P_{n}}\left(g, s, \Phi_{\varphi}\right)$ II: Mass-Jacquet-Shalika Eisenstein SERIES

Recall that when $\operatorname{Re}(s)>(n+1) / 2$, for $g \in \operatorname{Sp}_{n}\left(\mathbb{A}_{k}\right)$ and a section $\Phi \in I_{\mathbb{A}_{k}}(s)$ we defined

$$
E_{P_{n}}^{(r)}(g, s, \Phi)=\sum_{\gamma \in Q_{r}(k) \backslash \mathrm{GL}_{n}(k)} \Phi^{(r)}(\gamma g, s)
$$

where $\Phi^{(r)}(g, s)=M_{n, r}(s) \Phi(g, s) \in I_{n, r}(s)$. The discussion in Section 4.2 gives us the meromorphic continuation of $\Phi^{(r)}$. Moreover, for each $g \in \operatorname{Sp}_{n}\left(\mathbb{A}_{k}\right)$, the function $(a \mapsto$ $\left.E_{P_{n}}^{(r)}(\mathfrak{m}(a) g, s, \Phi)\right)$ can be viewed as a Mass-Jacquet-Shalika Eisenstein series on $\mathrm{GL}_{n}\left(\mathbb{A}_{k}\right)$. In this section, we recall the analytic behavior of this kind of Eisenstein series, and show that $E_{P_{n}}^{(r)}(\mathfrak{m}(a), s, \Phi)$ is holomorphic at $s=s_{(n)}$ for every $a \in \mathrm{GL}_{n}\left(\mathbb{A}_{k}\right)$ when $\Phi=\Phi_{\varphi}$ is a Siegel section.

Fix an integer $r$ with $0<r<n$. Set $X_{r}:=\operatorname{Mat}_{r \times n}$ (as an affine space over $k$ ). Let $\mu_{1}, \mu_{2}$ be two Hecke characters on $k^{\times} \backslash \mathbb{A}_{k}^{\times}$. For any $g \in \mathrm{GL}_{n}\left(\mathbb{A}_{k}\right)$ and Schwartz function 
$f \in S\left(X_{r}\left(\mathbb{A}_{k}\right)\right)$, define

$$
F(g)=F\left(g, \mu_{1}, \mu_{2}, f\right):=\mu_{1}(\operatorname{det} g)|\operatorname{det} g|_{\mathbb{A}_{k}}^{r / 2} \int_{\mathrm{GL}_{r}\left(\mathbb{A}_{k}\right)} f\left(h^{-1}\left(0, I_{r}\right) g\right) \mu^{-1}(\operatorname{det} h) d^{\times} h .
$$

Here $\mu=\mu_{1} \mu_{2}^{-1}|\cdot|_{\mathbb{A}_{k}}^{n / 2}$ and the Haar measure $d^{\times} h$ is normalized so that the volume of $\mathrm{GL}_{r}\left(O_{\mathbb{A}_{k}}\right)$ is 1 . This integral is absolutely convergent if $\left|\mu_{1} \mu_{2}^{-1}\right|=|\cdot|_{\mathbb{A}_{k}}^{\sigma}$ where $\sigma>r-n / 2$.

Recall that

$$
Q_{r}=\left\{\left(\begin{array}{cc}
a_{1} & * \\
0 & a_{2}
\end{array}\right) \in \mathrm{GL}_{n} \mid a_{1} \in \mathrm{GL}_{n-r}, a_{2} \in \mathrm{GL}_{r}\right\} .
$$

For $g \in \mathrm{GL}_{n}\left(\mathbb{A}_{k}\right)$ and $b=\left(\begin{array}{cc}a_{1} & * \\ 0 & a_{2}\end{array}\right) \in Q_{r}\left(\mathbb{A}_{k}\right)$, we have

$$
F(b g)=\mu_{1}\left(\operatorname{det} a_{1}\right) \mu_{2}\left(\operatorname{det} a_{2}\right) \delta_{Q_{r}}(b)^{1 / 2} F(g)
$$

where $\delta_{Q_{r}}(b)=\left|\operatorname{det} a_{1}\right|_{\mathbb{A}_{k}}^{r / 2} \cdot\left|\operatorname{det} a_{2}\right|_{\mathbb{A}_{k}}^{(r-n) / 2}$. The Maass-Jacquet-Shalika Eisenstein series associated to $f, \mu_{1}, \mu_{2}$ is defined by

$$
E\left(g, \mu_{1}, \mu_{2}, f\right)=\sum_{\gamma \in Q_{r}(k) \backslash \mathrm{GL}_{n}(k)} F(\gamma g) .
$$

This series converges absolutely when $\left|\mu_{1} \mu_{2}^{-1}\right|_{\mathbb{A}_{k}}=|\cdot|_{\mathbb{A}_{k}}^{\sigma}$ with $\sigma>n / 2$. The following theorem gives us the meromorphic continuation of such Eisenstein series (the proof is given in Theorem C.3):

Theorem 5.1. Suppose $\mu_{1} \cdot \mu_{2}^{-1}=|\cdot|_{\mathbb{A}_{k}}^{\sigma}$. Then

(1) (Continuation) $E\left(g, \mu_{1}, \mu_{2}, f\right)$ can be extended to a meromorphic function in $\sigma$ (in fact, a rational function in $\left.q^{-\sigma}\right)$, and every possible pole can only be a simple pole. Let $P(\sigma):=P^{+}(\sigma) \cdot P^{-}(\sigma)$, where

$$
P^{ \pm}(\sigma)=\prod_{i=0}^{r-1}\left(1-q^{-\sigma \pm\left(\frac{n}{2}-i\right)}\right) .
$$

Then $P(\sigma) \cdot E\left(g, \mu_{1}, \mu_{2}, f\right)$ is entire.

(2) (Functional equation) For each $f \in S\left(X_{r}\left(\mathbb{A}_{k}\right)\right)$, we have

$$
E\left(g, \mu_{1}, \mu_{2}, f\right)=E\left({ }^{t} g^{-1}, \mu_{1}^{-1}, \mu_{2}^{-1}, \hat{f}\right)
$$

where $\hat{f}$ is the Fourier transform of $f$ :

$$
\hat{f}(x):=\int_{X_{r}\left(\mathbb{A}_{k}\right)} f(y) \psi\left(-\operatorname{Tr}\left(x^{t} y\right)\right) d y .
$$

The Haar measure dy is chosen to be self-dual, i.e. $\hat{\hat{f}}(x)=f(-x)$.

(3) Suppose that there exists a place $v$ of $k$ such that the support of the restriction of $f$ on $X_{r}\left(k_{v}\right)$ is contained in the set of elements with rank $r$ in $X_{r}\left(k_{v}\right)$. Then

$$
P^{+}(\sigma) \cdot E\left(g, \mu_{1}, \mu_{2}, f\right) \text { is entire. }
$$

Now, we set

$$
\mu_{1, s}:=|\cdot|_{\mathbb{A}_{k}}^{s+\frac{n-r+1}{2}} \text { and } \mu_{2, s}:=|\cdot|_{\mathbb{A}_{k}}^{-s+\frac{r+1}{2}}
$$

Let $\widetilde{I}_{n, r}(s)$ be the space of smooth functions $\Psi$ on $\mathrm{GL}_{n}\left(\mathbb{A}_{k}\right)$ such that for every element $p=\left(\begin{array}{cc}a_{1} & * \\ 0 & a_{2}\end{array}\right) \in Q_{r}\left(\mathbb{A}_{k}\right)$ and $g \in \mathrm{GL}_{n}\left(\mathbb{A}_{k}\right)$,

$$
\Psi(p g)=\mu_{1, s}\left(\operatorname{det} a_{1}\right) \cdot \mu_{2, s}\left(\operatorname{det} a_{2}\right) \cdot\left(\left|\operatorname{det} a_{1}\right|_{\mathbb{A}_{k}}^{\frac{r}{2}} \cdot\left|\operatorname{det} a_{2}\right|_{\mathbb{A}_{k}}^{\frac{r-n}{2}}\right) \cdot \Psi(g) .
$$


FU-TSUN WEI

It is clear that $\widetilde{I}_{n, r}(s)=\otimes_{v}^{\prime} \widetilde{I}_{n, r, v}(s)$, and given $f=\otimes_{v} f_{v} \in S\left(X_{r}\left(\mathbb{A}_{k}\right)\right)=\otimes_{v}^{\prime} S\left(X_{r}\left(k_{v}\right)\right)$ we have,

$$
F\left(g, \mu_{1, s}, \mu_{2, s}, f\right)=\otimes_{v} F_{v}\left(g_{v}, \mu_{1, s, v}, \mu_{2, s, v}, f_{v}\right)
$$

for $g=\left(g_{v}\right)_{v} \in \mathrm{GL}_{n}\left(\mathbb{A}_{k}\right)$. Here

$$
F_{v}\left(g_{v}, \mu_{1, s, v}, \mu_{2, s, v}, f_{v}\right):=\mu_{1, s, v}\left(\operatorname{det} g_{v}\right)\left|\operatorname{det} g_{v}\right|_{v}^{r / 2} \int_{X_{r}\left(k_{v}\right)} f_{v}\left(h_{v}^{-1}\left(0, I_{r}\right) g_{v}\right) \mu_{s, v}^{-1}\left(h_{v}\right) d^{\times} h_{v}
$$

and $\mu_{s, v}=\mu_{1, s, v} \cdot \mu_{2, s, v}^{-1} \cdot|\cdot|_{v}^{n / 2}$. We have (cf. Lemma C.2)

Lemma 5.2. (1) For any standard section $\Psi_{v} \in \widetilde{I}_{n, r, v}(s)$, there exists a Schwartz function $f$ on $X_{r}\left(k_{v}\right)$ supported on elements of rank $r$ such that

$$
F_{v}\left(g_{v}, \mu_{1, v, s}, \mu_{2, v, s}, f_{v}\right)=\Psi_{v}\left(g_{v}, s\right) .
$$

(2) Let $f_{v}^{0}$ be the charateristic function of $X_{r}\left(O_{v}\right)$. Then

$$
F_{v}^{0}\left(g_{v}\right):=F_{v}\left(g, \mu_{1, s, v}, \mu_{2, s, v}, f_{v}^{0}\right)=\prod_{i=0}^{r-1} \zeta_{v}(2 s-2 n-r-i) \Psi_{v}^{0}
$$

where $\Psi_{v}^{0} \in \widetilde{I}_{n, r, v}(s)$ is the standard section such that

$$
\Psi_{v}^{0}\left(\kappa_{v}\right)=1, \forall \kappa_{v} \in \mathrm{GL}_{n}\left(O_{v}\right)
$$

For any $\Phi\left(\right.$ res. $\left.\Phi_{v}\right) \in I_{n, r}(s)$, we denote by $\widetilde{\Phi}$ (res. $\widetilde{\Phi}_{v}$ ) the restriction of $\Phi$ (res. $\Phi_{v}$ ) on $M_{n}\left(\mathbb{A}_{k}\right)\left(M_{n}\left(k_{v}\right)\right)$. Then via the isomorphism $\mathfrak{m}$ between $\mathrm{GL}_{n}$ and $M_{n}, \widetilde{\Phi}$ (res. $\widetilde{\Phi}_{v}$ ) can be viewed as a function in $\widetilde{I}_{n, r}(s)$ (res. $\left.\widetilde{I}_{n, r, v}(s)\right)$. Let $c_{r}(s):=\prod_{v} c_{r, v}(s)$ where

$$
c_{r, v}(s):=\prod_{i=0}^{r-1} \zeta_{k, v}(2 s+n-r-i) .
$$

For each factorizable Schwartz function $\varphi=\otimes_{v} \varphi_{v} \in S\left(V\left(\mathbb{A}_{k}\right)^{n}\right)$, from the discussion in Section 4.2 and Lemma 5.2 we get

$$
\begin{aligned}
& \widetilde{M_{n, r}(s)} \Phi_{\varphi} \\
& =\frac{a_{r}\left(s^{\prime}\right)}{b_{r}\left(s^{\prime}\right)} \cdot\left(\left(\otimes_{v \notin \Sigma\left(\Phi_{\varphi}, d n\right)} \Psi_{v}^{0}\right) \otimes\left(\otimes_{v \in \Sigma\left(\Phi_{\varphi}, d n\right)} \frac{b_{r, v}\left(s^{\prime}\right)}{a_{r, v}\left(s^{\prime}\right)} M_{n, r, v}(s) \Phi_{\varphi_{v}}\right)\right) \\
& =\frac{a_{r}\left(s^{\prime}\right)}{c_{r}(s) b_{r}\left(s^{\prime}\right)} \cdot\left(F^{0, \varphi} \otimes\left(\otimes_{v \in \Sigma\left(\Phi_{\varphi}, d n\right)} \frac{c_{r, v}(s) b_{r, v}\left(s^{\prime}\right)}{a_{r, v}\left(s^{\prime}\right)} M_{n, r, v}(s) \Phi_{\varphi_{v}}\right)\right),
\end{aligned}
$$

where $s^{\prime}=s+(r-n) / 2, \Sigma(\Phi, d n)$ is the finite set of places $v$ of $k$ such that $\Phi_{v} \neq \Phi_{v}^{0}$ or $\operatorname{vol}\left(N_{n}\left(O_{v}\right)\right) \neq 1$, and $F^{0, \varphi}=\otimes_{v \notin \Sigma\left(\Phi_{\varphi}, d n\right)} F_{v}^{0}$.

Lemma 5.3. For any $\varphi_{v} \in S\left(V\left(k_{v}\right)^{n}\right)$, there exists a finite collection of standard sections $\Phi_{v}^{j}$ in $I_{v}(s)$ such that

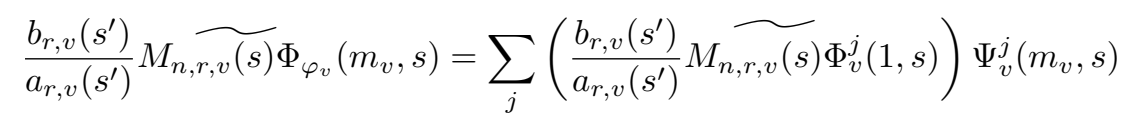

for all $m_{v} \in M_{n}\left(k_{v}\right)$, where $\Psi_{v}^{j} \in \widetilde{I}_{n, r, v}(s)$ is a standard section for each $j$.

Then Lemma 5.2 and 5.3 lead us to the following result:

Proposition 5.4. (1) For each Siegel section $\Phi_{\varphi_{v}} \in I_{v}(s)$, there exists a finite collection of Schwartz functions $f_{v, j} \in S\left(X_{r}\left(k_{v}\right)\right)$ such that

$$
\frac{c_{r, v}(s) b_{r, v}\left(s^{\prime}\right)}{a_{r, v}\left(s^{\prime}\right)} M_{n, r, v} \widetilde{(s)} \Phi_{\varphi_{v}}=\sum_{j} \beta_{v, j} F_{v, j}
$$


where for each $j, \beta_{v, j}$ is a rational function in $q^{-s}$ which is holomorphic at $s=s_{(n)}$, and

$$
F_{v, j}\left(g_{v}\right)=F\left(g_{v}, \mu_{1, s, v}, \mu_{2, s, v}, f_{v, j}\right) \text {, for } g_{v} \in \mathrm{GL}_{n}\left(k_{v}\right) \text {. }
$$

(2) Moreover, suppose $a_{r, v}\left(s^{\prime}\right)^{-1} M_{n, r, v}(s) \Phi_{\varphi_{v}}\left(g_{v}, s\right)$ has a zero at $s=s_{(n)}$ for all elements $g_{v} \in \operatorname{Sp}_{n}\left(k_{v}\right)$. Then we are able to choose suitable $f_{v, j}$ and $\beta_{v, j}$ such that either $\beta_{v, j}$ has a zero at $s=s_{(n)}$ for all $j$ or the support of $f_{v, j}$ is contained in the set of elements of rank $r$ in $X_{r}\left(k_{v}\right)$ for all $j$.

The above proposition describes the analytic behavior of the local factors at each place $v \in \Sigma\left(\Phi_{\varphi}, d n\right)$. Immediately, we have

Corollary 5.5. For any factorizable $\varphi=\otimes_{v} \varphi_{v} \in S\left(V\left(\mathbb{A}_{k}\right)^{n}\right)$, there exists a finite collection of Schwartz functions $f_{j} \in S\left(X_{r}\left(\mathbb{A}_{k}\right)\right)$ such that

$$
\widetilde{\Phi}_{\varphi}^{(r)}=\widetilde{M_{n, r}(s)} \Phi_{\varphi}=\frac{a_{r}\left(s^{\prime}\right)}{c_{r}(s) b_{r}\left(s^{\prime}\right)} \sum_{j} \beta_{j}(s) F_{j}(s)
$$

where for each $j, \beta_{j}$ is a rational function in $q^{-s}$ which is holomorphic at $s_{(n)}$, and $F^{j}$ is the section of $\widetilde{I}_{n, r}(s)$ corresponding to $f_{j}$.

5.1. The order of $E_{P_{n}}^{(r)}\left(\mathfrak{m}(a), s, \Phi_{\varphi}\right)$ at $s=s_{(n)}$ for $0<r<n$. By Corollary 5.5 we have that for $a \in \mathrm{GL}_{2}\left(\mathbb{A}_{k}\right)$,

$$
E_{P_{n}}^{(r)}\left(\mathfrak{m}(a), s, \Phi_{\varphi}\right)=\frac{a_{r}\left(s^{\prime}\right)}{c_{r}(s) b_{r}\left(s^{\prime}\right)} \cdot \sum_{j} \beta_{j}(s) \cdot E\left(a, \mu_{1, s}, \mu_{2, s}, f_{j}\right) .
$$

Recall that $s_{(n)}=\operatorname{dim}(V) / 2-(n+1) / 2, s^{\prime}=s+(n-r) / 2$, and

$$
\begin{aligned}
& a_{r}(s)=L\left(s+\frac{r+1}{2}-r, \chi_{V}\right) \prod_{i=1}^{\lfloor r / 2\rfloor} \zeta_{k}(2 s-r+2 i), \\
& b_{r}(s)=L\left(s+\frac{r+1}{2}, \chi_{V}\right) \prod_{i=1}^{\lfloor r / 2\rfloor} \zeta_{k}(2 s+r-2 i+1), \\
& c_{r}(s)=\prod_{i=0}^{r-1} \zeta_{k}(2 s+n-r-i) .
\end{aligned}
$$

Therefore

$$
\operatorname{ord}_{s=s_{(n)}} c_{r}(s)^{-1}= \begin{cases}+2, & \text { if } \operatorname{dim}(V)=4 \text { and } r=2 \\ +1, & \text { if }(\operatorname{dim}(V), r)=(2,1) \text { or }(4,3), \\ 0, & \text { otherwise. }\end{cases}
$$

By Lemma 4.2 we have

$$
\operatorname{ord}_{s=s_{(n)}} \frac{a_{r}\left(s^{\prime}\right)}{c_{r}(s) b_{r}\left(s^{\prime}\right)}= \begin{cases}+1, & \text { if } \operatorname{dim}(V)=2, \\ -1, & \text { if }(\operatorname{dim}(V), r)=(4,1) \text { and } \chi_{V} \text { is trivial, } \\ 0, & \text { if }(\operatorname{dim}(V), r)=(4,1) \text { and } \chi_{V} \text { is non-trivial, } \\ 0, & \text { if }(\operatorname{dim}(V), r)=(4,2) \text { and } \chi_{V} \text { is trivial, } \\ +1, & \text { if }(\operatorname{dim}(V), r)=(4,2) \text { and } \chi_{V} \text { is non-trivial, } \\ +1, & \text { if } \operatorname{dim}(V)=4 \text { and } r \geq 3\end{cases}
$$

Moreover, by Theorem 5.1,

$$
\operatorname{ord}_{s=s_{(n)}} E\left(a, \mu_{1, s}, \mu_{2, s}, f_{j}\right) \geq \begin{cases}-1 & \text { if }(\operatorname{dim}(V), r)=(2,1),(4,2), \text { or }(4,3), \\ -1 & \text { if } n=2 \text { and }(\operatorname{dim}(V), r)=(4,1) \\ 0 & \text { otherwise. }\end{cases}
$$


Suppose $\operatorname{dim}(V)=2$. Then $E_{P_{n}}^{(r)}\left(\mathfrak{m}(a), s, \Phi_{\varphi}\right)$ is holomorphic at $s=s_{(n)}$ and vanishes when $r>1$. Suppose $\operatorname{dim}(V)=4$. We have that $E_{P_{n}}^{(r)}\left(\mathfrak{m}(a), s, \Phi_{\varphi}\right)$ is holomorphic (resp. vanishes) at $s=s_{(n)}$ when $r=3$ (resp. $r>3$ ). Suppose $r=1$ or 2 . Since $\left(V, Q_{V}\right)$ is anisotropic over $k$, there exists at least one (resp. two) place $v$ of $k$ such that $\left(V, Q_{V}\right)$ is still anisotropic over $k_{v}$ when $\operatorname{dim}(V)=4$ and $\chi_{V}$ is trivial (resp. other cases). Therefore by Lemma 4.4 (ii) and Proposition 5.4 (ii), we always can choose $\beta_{j}$ such that

$$
\operatorname{ord}_{s=s_{(n)}} \beta_{j} \geq 1 \quad \text { (resp. 2). }
$$

Therefore $E_{P_{n}}^{(r)}\left(\mathfrak{m}(a), s, \Phi_{\varphi}\right)$ is still holomorphic at $s_{(n)}$ and vanishes for the case when $r=2$ or $n-1>r=1$. We conclude that

Proposition 5.6. Fix an integer $r$ with $0<r<n$. For each $a \in \mathrm{GL}_{n}\left(\mathbb{A}_{k}\right), E_{P_{n}}^{(r)}\left(\mathfrak{m}(a), s, \Phi_{\varphi}\right)$ is holomorphic at $s=s_{(n)}$. Moreover, $E_{P_{n}}^{(r)}\left(\mathfrak{m}(a), s_{(n)}, \Phi_{\varphi}\right)=0$ except for the following cases:

(i) when $\operatorname{dim}(V)=2, r=1$;

(ii) when $\operatorname{dim}(V)=4, n=2=r+1$;

(iii) when $\operatorname{dim}(V)=4, r=3$.

Together with the result in Proposition 4.6, we obtain:

Corollary 5.7. For $0 \leq r \leq n, E_{P_{n}}^{(r)}\left(\mathfrak{m}(a), s_{(n)}, \Phi_{\varphi}\right)=0$ except for the following cases:

(i) when $r=0$;

(ii) when $\operatorname{dim}(V)=2, n \geq r=1$;

(iii) when $\operatorname{dim}(V)=4, n=2 \geq r \geq 1$;

(iv) when $\operatorname{dim}(V)=4, n \geq r=3$.

\section{Siegel-Weil formula}

The aim of this section is to prove the Siegel-Weil formula over function fields:

Theorem 6.1. Let $\varphi \in S\left(V\left(\mathbb{A}_{k}\right)^{n}\right)$. Then for $g \in \operatorname{Sp}_{n}\left(\mathbb{A}_{k}\right)$,

$$
E\left(g, s_{(n)}, \Phi_{\varphi}\right)=\epsilon(n) \cdot I(g, \varphi),
$$

where $s_{(n)}=\operatorname{dim}(V) / 2-(n+1) / 2$ and

$$
\epsilon(n)= \begin{cases}1 & \text { if } \operatorname{dim}(V)>n+1, \\ 2 & \text { if } \operatorname{dim}(V) \leq n+1\end{cases}
$$

The proof is divided into three cases:

(i) $\operatorname{dim}(V)=n+1$;

(ii) $\operatorname{dim}(V)>n+1$;

(iii) $\operatorname{dim}(V)<n+1$.

We deal with these cases in Section 6.1, 6.2, and 6.3, separately.

6.1. Special case: $\operatorname{dim}(V)=n+1$. We first show that

Lemma 6.2. When $\operatorname{dim}(V)=n+1$,

$$
M(0) \Phi_{\varphi}(g, 0)=\Phi_{\varphi}(g, 0)
$$

for every Siegel section $\Phi_{\varphi}$ and $g \in \operatorname{Sp}_{n}\left(\mathbb{A}_{k}\right)$ when $\operatorname{dim}(V)=n+1$. In particular, for every $a \in \mathrm{GL}_{n}\left(\mathbb{A}_{k}\right)$,

$$
E_{P_{n}}^{(n)}\left(\mathfrak{m}(a), 0, \Phi_{\varphi}\right)=\left(M(0) \Phi_{\varphi}\right)(\mathfrak{m}(a), 0)=\Phi_{\varphi}(\mathfrak{m}(a), 0)=(\omega(\mathfrak{m}(a)) \varphi)(0) .
$$


Proof. For each place $v$ of $k$, the maps

$$
T_{1, v}=\left(\varphi_{v} \mapsto \Phi_{\varphi}(\cdot, 0)\right) \quad \text { and } \quad T_{2, v}=\left(\varphi_{v} \mapsto \frac{b_{n, v}(0)}{a_{n, v}(0)} M_{v}(0) \Phi_{\varphi}(\cdot, 0)\right)
$$

are both lie in $\operatorname{Hom}_{\mathrm{Sp}_{n}\left(k_{v}\right) \times \varnothing(V)\left(k_{v}\right)}\left(S\left(V\left(k_{v}\right)^{n}\right), I_{\mathbb{A}_{k}}(0) \otimes \mathbf{1}\right)$. Lemma 4.4 implies that there exists $\mu_{v} \in \mathbb{C}$ such that $T_{2, v}=\mu_{v} T_{1, v}$. Recall that for every $\Phi=\otimes_{v} \Phi_{v} \in I_{\mathbb{A}_{k}}(s), M(s) \Phi(g, s)$ can be expressed by

$$
\frac{a_{n}(s)}{b_{n}(s)} \cdot\left[\left(\otimes_{v \notin \Sigma(\Phi, d n)} \Phi_{v}^{0}\left(g_{v},-s\right)\right) \otimes\left(\otimes_{v \in \Sigma(\Phi, d n)} \frac{b_{n, v}(s)}{a_{n, v}(s)} M_{v}(s) \Phi_{v}\left(g_{v}, s\right)\right)\right] .
$$

Hence we can find $\mu \in \mathbb{C}$ such that $M(0) \Phi_{\varphi}(\cdot, 0)=\mu \Phi_{\varphi}(\cdot, 0)$ for every $\varphi \in S\left(V\left(\mathbb{A}_{k}\right)^{n}\right)$. It is known that $M(0) \circ M(0): I_{\mathbb{A}_{k}}(0) \rightarrow I_{\mathbb{A}_{k}}(0)$ is the identity map. Thus $\mu= \pm 1$. By Theorem A.4, we can choose a Siegel section $\Phi_{\varphi}$ such that the Siegel-Eisenstein series $E\left(\cdot, 0, \Phi_{\varphi}\right)$ is not zero. Its constant term $E_{P_{n}}\left(g, s, \Phi_{\varphi}\right)$ is equal to

$$
\Phi_{\varphi}(g, 0)+M(0) \Phi_{\varphi}(g, 0)=(1+\mu)(\omega(g) \varphi)(0) .
$$

Suppose $\mu=-1$. Then $E_{P_{n}}\left(\cdot, 0, \Phi_{\varphi}\right) \equiv 0$. By Corollary 3.3 we have $E\left(\cdot, 0, \Phi_{\varphi}\right) \equiv 0$, which gives us a contradiction. Therefore $\mu=1$ and the proof is complete.

Corollary 6.3. The Siegel-Weil formula (Theorem 6.1) holds when $\operatorname{dim}(V)=n+1$.

Proof. It suffices to show that for $\varphi \in S\left(V\left(\mathbb{A}_{k}\right)^{n}\right)$ and $g \in \operatorname{Sp}_{n}\left(\mathbb{A}_{k}\right)$,

$$
E_{B_{n}}\left(g, s_{(n)}, \Phi_{\varphi}\right)=2 \cdot I_{B_{n}}(g, \varphi) .
$$

Indeed, Proposition 2.3 and Proposition 3.2 say that $E\left(g, s_{(n)}, \Phi_{\varphi}\right)$ and $I(g, \varphi)$ are both concentrated on the Borel subgroup $B_{n}$. Then by $(6.1)$, it can be shown that $E\left(\cdot, s_{(n)}, \Phi_{\varphi}\right)-$ $2 I(\cdot, \varphi)$ is a cusp form on $\operatorname{Sp}_{n}\left(\mathbb{A}_{k}\right)$ which is also orthogonal to all the cusp forms on $\operatorname{Sp}_{n}\left(\mathbb{A}_{k}\right)$. Therefore the result holds.

Note that

and

$$
E_{B_{n}}\left(g, s_{(n)}, \Phi_{\varphi}\right)=\int_{U_{M_{n}}(k) \backslash U_{M_{n}}\left(\mathbb{A}_{k}\right)} E_{P_{n}}\left(u g, s_{(n)}, \Phi_{\varphi}\right) d u
$$

$$
I_{B_{n}}(g, \varphi)=\int_{U_{M_{n}}(k) \backslash U_{M_{n}}\left(\mathbb{A}_{k}\right)} I_{P_{n}}(u g, \varphi) d u
$$

where $U_{M_{n}}:=U_{n} \cap M_{n}$. Write $g=n(b) m(a) \kappa$ where $\mathfrak{n}(b) \in N_{n}\left(\mathbb{A}_{k}\right), \mathfrak{m}(a) \in M_{n}\left(\mathbb{A}_{k}\right)$, $\kappa \in \operatorname{Sp}_{n}\left(O_{\mathbb{A}_{k}}\right)$, then

$$
E_{P_{n}}\left(g, s_{(n)}, \Phi_{\varphi}\right)=E_{P_{n}}\left(\mathfrak{m}(a), s_{(n)}, \Phi_{\omega(\kappa) \varphi}\right)
$$

and

$$
(\omega(g) \varphi)(0)=\omega(\mathfrak{m}(a))(\omega(\kappa) \varphi)(0) .
$$

To show (6.1), it is enough to prove that for every $\varphi \in S\left(V\left(\mathbb{A}_{k}\right)^{n}\right)$ and $a \in \mathrm{GL}_{n}\left(\mathbb{A}_{k}\right)$,

$$
E_{P_{n}}\left(\mathfrak{m}(a), s_{(n)}, \Phi_{\varphi}\right)=\Phi_{\varphi}(\mathfrak{m}(a), 0)+M(0) \Phi_{\varphi}(\mathfrak{m}(a), 0)=2(\omega(\mathfrak{m}(a)) \varphi)(0) .
$$

Therefore Lemma 6.2 completes the proof.

6.2. Special case: $\operatorname{dim}(V)>n+1$. Let $\varphi_{0} \in S\left(V\left(\mathbb{A}_{k}\right)\right)$ such that $\varphi_{0}(0)=1$. For every $\varphi \in S\left(V\left(\mathbb{A}_{k}\right)^{n}\right)$, take $\tilde{\varphi}:=\varphi_{0} \otimes \varphi \in S\left(V\left(\mathbb{A}_{k}\right)^{n+1}\right)$. Then it is clear that for $g \in \operatorname{Sp}_{n}\left(\mathbb{A}_{k}\right)$,

$$
\left(i^{*} \Phi_{\tilde{\varphi}}\right)(g, s)=\Phi_{\tilde{\varphi}}(i(g), s-1 / 2)=\Phi_{\varphi}(g, s),
$$

where $i: \mathrm{Sp}_{n} \hookrightarrow \mathrm{Sp}_{n+1}$ is the embedding introduced in Section 4.2. Consider the maximal standard parabolic subgroup $P_{1}$ of $\mathrm{Sp}_{n+1}$ whose Levi subgroup $M_{1}$ is isomorphic to $\mathrm{GL}_{1} \times \mathrm{Sp}_{n}$. By Lemma 3.1, we have 
Lemma 6.4. For every standard section $\Phi \in I_{\mathbb{A}_{k}}^{n+1}(s)$ and $g \in \operatorname{Sp}_{n}\left(\mathbb{A}_{k}\right)$,

$$
E_{P_{1}}^{n+1}(i(g), s, \Phi)=E^{n}\left(g, s+\frac{1}{2}, i^{*} \Phi\right)+E^{n}\left(g, \frac{1}{2}-s, i^{*} M_{n+1}(s) \Phi\right) .
$$

Proof. By Lemma 3.1, we get

$$
E_{P_{1}}^{n+1}(i(g), s, \Phi)=E^{n}\left(g, s+\frac{1}{2}, i^{*} \Phi\right)+E^{n}\left(g, s-\frac{1}{2}, i^{*} \Phi_{w}\right) .
$$

where

$$
w=w_{n+1} w_{n}^{-1}=\left(\begin{array}{cccc}
0 & 0 & 1 & 0 \\
0 & I_{n} & 0 & 0 \\
-1 & 0 & 0 & 0 \\
0 & 0 & 0 & I_{n}
\end{array}\right) \in \operatorname{Sp}_{n+1}(k) .
$$

Recall the functional equation of Siegel-Eisenstein series:

$$
E^{n}(g, s, \Phi)=E^{n}(g,-s, M(s) \Phi), \quad \forall g \in \operatorname{Sp}_{n}\left(\mathbb{A}_{k}\right), \Phi \in I_{\mathbb{A}_{k}}^{n}(s) .
$$

By straightforward calculation, $M_{n}(s-1 / 2)\left(i^{*} \Phi_{w}\right)=i^{*}\left(M_{n+1}(s) \Phi\right)$. Therefore the result holds.

When $\operatorname{dim}(V)=4$ and $n=2$, by Lemma 6.2 , it can be observed that for every $g \in \operatorname{Sp}_{2}\left(\mathbb{A}_{k}\right)$

$$
E^{2}\left(g, \frac{1}{2}, i^{*} M_{3}(0) \Phi_{\tilde{\varphi}}\right)=E^{2}\left(g, \frac{1}{2}, i^{*} \Phi_{\tilde{\varphi}}\right)
$$

In particular,

$$
E_{P_{1}}^{3}\left(i(g), 0, \Phi_{\tilde{\varphi}}\right)=2 E^{2}\left(g, \frac{1}{2}, i^{*} \Phi_{\tilde{\varphi}}\right)=2 E^{2}\left(g, \frac{1}{2}, \Phi_{\varphi}\right) .
$$

On the other hand, by Corollary 6.3 we get

$$
E_{P_{1}}^{3}\left(i(g), 0, \Phi_{\tilde{\varphi}}\right)=2 I_{P_{1}}^{3}(i(g), \tilde{\varphi})=2 I^{2}(g, \varphi) .
$$

Therefore

Corollary 6.5. The Siegel-Weil formula (Theorem 6.1) holds when $\operatorname{dim}(V)=4$ and $n=2$.

When $\operatorname{dim}(V)=4$ and $n=1$, by the same argument in Corollary 6.3, it is enough to show that for $a \in \mathrm{GL}_{1}\left(\mathbb{A}_{k}\right)$

$$
E_{P_{1}}\left(\mathfrak{m}(a), 0, \Phi_{\varphi}\right)=(\omega(\mathfrak{m}(a)) \varphi)(0) .
$$

By Corollary 5.7 we have $E_{P_{1}}^{(1)}\left(\mathfrak{m}(a), 0, \Phi_{\varphi}\right)=0$. Hence

$$
E_{P_{1}}\left(\mathfrak{m}(a), 0, \Phi_{\varphi}\right)=E_{P_{1}}^{(0)}\left(\mathfrak{m}(a), 0, \Phi_{\varphi}\right)=(\omega(\mathfrak{m}(a)) \varphi)(0) .
$$

We conclude that

Corollary 6.6. The Siegel-Weil formula (Theorem 6.1) holds when $\operatorname{dim}(V)>n+1$.

6.3. Special case: $\operatorname{dim}(V)<n+1$. Set $\ell:=n+1-\operatorname{dim}(V)$. The case when $\ell=0$ was shown in Corollary 6.3. The remaining case is proven by an induction process on $\ell$.

By the same argument in Corollary 6.3, we only need to show that for $a \in \mathrm{GL}_{n}\left(\mathbb{A}_{k}\right)$

$$
E_{P_{n}}\left(\mathfrak{m}(a), s_{(n)}, \Phi_{\varphi}\right)=2(\omega(\mathfrak{m}(a)) \varphi)(0) .
$$

Note that when $\operatorname{dim}(V) \leq n+1$, by Corollary 5.7 we have that for $a \in \mathrm{GL}_{n}\left(\mathbb{A}_{k}\right)$

$$
E_{P_{n}}\left(\mathfrak{m}(a), s_{(n)}, \Phi_{\varphi}\right)=\Phi_{\varphi}\left(\mathfrak{m}(a), s_{(n)}\right)+E_{P_{n}}^{(\operatorname{dim}(V)-1)}\left(\mathfrak{m}(a), s_{(n)}, \Phi_{\varphi}\right) .
$$

Then it is clear that $\left(a \mapsto E_{P_{n}}\left(\mathfrak{m}(a), s_{(n)}, \Phi_{\varphi}\right)\right)$ is concentrated on the Borel subgroup of

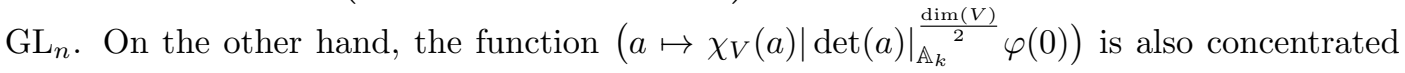


on the Borel subgroup of $\mathrm{GL}_{n}$. Therefore it is enough to show the equality for their constant terms (along $Q_{n-1}$ ):

$$
\left(E_{P_{n}}\right)_{Q_{n-1}}\left(\mathfrak{m}(a), s_{(n)}, \Phi_{\varphi}\right)=2 \chi_{V}(a)|\operatorname{det}(a)|_{\mathbb{A}_{k}{ }^{2}}^{\frac{\operatorname{dim}(V)}{2}} \varphi(0) .
$$

Here $Q_{r}, 0<r<n$, is the maximal parabolic subgroup of $\mathrm{GL}_{n}$ introduced in Section 5 . Without loss of generality, we only need to consider the case when

$$
a \in \mathrm{GL}_{n-1}\left(\mathbb{A}_{k}\right) \hookrightarrow \mathrm{GL}_{1}\left(\mathbb{A}_{k}\right) \times \mathrm{GL}_{n-1}\left(\mathbb{A}_{k}\right) .
$$

Note that $Q_{n-1} \subset P_{1} \cap P_{n}$, and

$$
\left(E_{P_{n}}\right)_{Q_{n-1}}\left(\mathfrak{m}(a), s_{(n)}, \Phi_{\varphi}\right)=\left(E_{P_{1}}\right)_{P_{n-1}^{n-1}}\left(\mathfrak{m}(a), s_{(n)}, \Phi_{\varphi}\right) .
$$

Here $P_{n-1}^{n-1}$ is the Siegel parabolic subgroup of $\mathrm{Sp}_{n-1}$. By Lemma 6.4, we have that for $a \in \mathrm{GL}_{n-1}\left(\mathbb{A}_{k}\right)$.

$$
E_{P_{1}}\left(\mathfrak{m}(a), s_{(n)}, \Phi_{\varphi}\right)=E^{n-1}\left(\mathfrak{m}(a), s_{(n-1)}, i^{*} \Phi_{\varphi}\right)+E^{n-1}\left(\mathfrak{m}(a), \frac{1}{2}-s_{(n)}, i^{*} M_{n}\left(s_{(n)}\right) \Phi_{\varphi}\right) .
$$

Since $M_{n}(s) \Phi_{\varphi} \equiv 0$ when $s=s_{(n)}$ and $\ell>0$, it is observed that for $a \in \mathrm{GL}_{n-1}\left(\mathbb{A}_{k}\right)$,

$$
E^{n-1}\left(\mathfrak{m}(a), \frac{1}{2}-s_{(n)}, i^{*} M_{n}\left(s_{(n)}\right) \Phi_{\varphi}\right)=0 .
$$

By induction we have that for $a \in \mathrm{GL}_{n-1}\left(\mathbb{A}_{k}\right)$,

$$
\left(E_{P_{n}}\right)_{Q_{n-1}}\left(\mathfrak{m}(a), s_{(n)}, \Phi_{\varphi}\right)=\left(E_{P_{1}}\right)_{P_{n-1}^{n-1}}\left(\mathfrak{m}(a), s_{(n)}, \Phi_{\varphi}\right)=2 \chi_{V}(a)|\operatorname{det}(a)|_{\mathbb{A}_{k}}^{\frac{\operatorname{dim}(V)}{2}} \varphi(0) .
$$

Therefore we conclude that

Corollary 6.7. The Siegel-Weil formula (Theorem 6.1) holds when $\operatorname{dim}(V)<n+1$.

\section{Appendix A. Fourier COEFFicients of theta SERIES}

Let $f$ be an automorphic form on $\operatorname{Sp}_{n}\left(\mathbb{A}_{k}\right)$. For each $\beta \in \operatorname{Sym}_{n}(k)$, the $\beta$-th Fourier coefficient of $f$ is

$$
f_{\beta}(g):=\int_{\operatorname{Sym}_{n}(k) \backslash \operatorname{Sym}_{n}\left(\mathbb{A}_{k}\right)} f(\mathfrak{n}(b) g) \psi(\operatorname{Tr}(-b \beta)) d b,
$$

where $\operatorname{Tr}$ is the trace map and the Haar measure $d b$ is normalized so that the total mass is 1 . The aim of this section is to compare $E_{\beta}\left(g, s_{(n)}, \varphi\right)$ with $I_{\beta}^{n}(g, \varphi)$ when $\operatorname{det} \beta \neq 0$, and prove an analogous result (Theorem A.5) of Proposition 4.2 in [15] by the same strategy.

It is clear that

$$
\begin{aligned}
I_{\beta}^{n}(g, \varphi) & =\int_{\operatorname{Sym}_{n}(k) \backslash \operatorname{Sym}_{n}\left(\mathbb{A}_{k}\right)} I(\mathfrak{n}(b) g, \varphi) \psi(\operatorname{Tr}(-b \beta)) d b \\
& =\int_{\mathrm{O}(V)(k) \backslash \mathrm{O}(V)\left(\mathbb{A}_{k}\right)} \sum_{\substack{x \in V(k) n \\
Q_{V}^{(n)}(x)=\beta}} \omega(g) \varphi\left(h^{-1} x\right) d h .
\end{aligned}
$$

Here $Q_{V}^{(n)}$ is the moment map from $V^{n}$ to $\operatorname{Sym}_{n}$ introduced in Section 1.3. Thus $I_{\beta}^{n}(g, \varphi)=0$ if $\left(Q_{V}^{(n)}\right)^{-1}(\beta)$ is empty.

Recall that a gauge form on a given smooth variety $\mathcal{V}$ over $k$ is a differential $\nu$-form over $k$ (where $\nu=\operatorname{dim}(\mathcal{V})$ ) which is regular and non-vanishing everywhere. We refer the reader to [20] for further discussions of the gauge forms on varieties. Let $d x$ and $d b$ be the standard gauge forms on the vector spaces $V^{n}$ and $\mathrm{Sym}_{n}$ over $k$, respectively. The corresponding measures (i.e. Tamagawa measures) on $V^{n}\left(\mathbb{A}_{k}\right)$ and $\operatorname{Sym}_{n}\left(\mathbb{A}_{k}\right)\left(\right.$ resp. $V^{n}\left(k_{v}\right)$ and $\left.\operatorname{Sym}_{n}\left(k_{v}\right)\right)$ 
are also denoted by $d x$ and $d b\left(\right.$ resp. $d x_{v}$ and $\left.d b_{v}\right)$. For every $\beta$ in $\operatorname{Sym}_{n}(k)\left(\operatorname{resp} . \operatorname{in} \operatorname{Sym}_{n}\left(k_{v}\right)\right)$, let

$$
\left(Q_{V}^{(n)}\right)_{\mathrm{reg}}^{-1}(\beta):=\left\{x \in\left(Q_{V}^{(n)}\right)^{-1}(\beta) \mid d Q_{V}^{(n)}(x): V^{n} \rightarrow \operatorname{Sym}_{n} \text { is sujective }\right\},
$$

which is a smooth variety over $k$ (resp. $k_{v}$ ), and there exists a gauge form $\delta_{\beta}$ on $\left(Q_{V}^{(n)}\right)_{\text {reg }}^{-1}(\beta)$ which is compatible with the choice of $d x$ and $d b$ (resp. $d x_{v}$ and $d b_{v}$ ) on $V^{n}$ and $\operatorname{Sym}_{n}$ respectively. In concrete terms, $\delta_{\beta}$ induces a local measure on $\left(Q_{V}^{(n)}\right)^{-1}(\beta)\left(k_{v}\right)$ so that for any $L^{1}$-function $f_{v}$ on $V\left(k_{v}\right)^{n}$ (cf. [19] $\S 6$ ),

$$
\int_{V\left(k_{v}\right)^{n}} f_{v} d x_{v}=\int_{\operatorname{Sym}_{n}\left(k_{v}\right)}\left(\int_{\left(Q_{V}^{(n)}\right)_{\text {reg }}^{-1}\left(b_{v}\right)\left(k_{v}\right)} f_{v} \delta_{b_{v}}\right) d b_{v} .
$$

In particular, suppose $\beta \in \operatorname{Sym}_{n}(k)$ with $\operatorname{det} \beta \neq 0$ and $\left(Q_{V}^{(n)}\right)^{-1}(\beta)(k)$ is not empty, which implies that $\operatorname{dim}(V) \geq n$. Then

$$
\left(Q_{V}^{(n)}\right)_{\mathrm{reg}}^{-1}(\beta)=\left(Q_{V}^{(n)}\right)^{-1}(\beta)
$$

and $\mathrm{O}(V)$ acts transitively on $\left(Q_{V}^{(n)}\right)^{-1}(\beta)$. Take any element $x \in\left(Q_{V}^{(n)}\right)^{-1}(\beta)$. Let

$$
\mathrm{St}_{\mathrm{O}(V)}(x):=\{h \in \mathrm{O}(V): h x=x\} .
$$

Then $\mathrm{St}_{\mathrm{O}(V)}(x) \backslash \mathrm{O}(V)$ is isomorphic (as a variety over $k$ ) to $\left(Q_{V}^{(n)}\right)^{-1}(\beta)$ by

$$
h \longmapsto h^{-1} x .
$$

We take the measure $\tilde{d} h$ on $\mathrm{St}_{\mathrm{O}(V)}(x)\left(\mathbb{A}_{k}\right) \backslash \mathrm{O}(V)\left(\mathbb{A}_{k}\right)$ to be the measure on $\left(Q_{V}^{(n)}\right)^{-1}(\beta)\left(\mathbb{A}_{k}\right)$ determined by the gauge form $\delta_{\beta}$, with a set $\left\{\lambda_{v}(\beta)\right\}_{v}$ of convergence factors. The measures $d h$ on $\mathrm{O}(V)\left(\mathbb{A}_{k}\right)$ and $\tilde{d} h$ on $\mathrm{St}_{\mathrm{O}(V)}(x)\left(\mathbb{A}_{k}\right) \backslash \mathrm{O}(V)\left(\mathbb{A}_{k}\right)$ induce a unique measure $d h^{\prime}$ on $\mathrm{St}_{\mathrm{O}(V)}(x)\left(\mathbb{A}_{k}\right)$ such that

$$
d h=d h^{\prime} \tilde{d} h .
$$

Then we can write

$$
I_{\beta}(g, \varphi)=\operatorname{vol}\left(\operatorname{St}_{\mathrm{O}(V)}(x)(k) \backslash \operatorname{St}_{\mathrm{O}(V)}\left(\mathbb{A}_{k}\right), d h^{\prime}\right) \cdot \int_{\mathrm{St}_{\mathrm{O}(V)}(x)\left(\mathbb{A}_{k}\right) \backslash \mathrm{O}(V)\left(\mathbb{A}_{k}\right)} \omega(g) \varphi\left(h^{-1} x\right) \tilde{d} h .
$$

When $\varphi$ is a pure tensor, say $\varphi=\otimes_{v} \varphi_{v}$, write $\tilde{d} h$ as $\prod_{v} \lambda_{v}(\beta)^{-1} \tilde{d} h_{v}$ we get

$$
\begin{aligned}
I_{\beta}(g, \varphi)= & \operatorname{vol}\left(\operatorname{St}_{\mathrm{O}(V)}(x)(k) \backslash \mathrm{St}_{\mathrm{O}(V)}\left(\mathbb{A}_{k}\right), d h^{\prime}\right) \\
& \cdot \prod_{v}\left(\lambda_{v}(\beta)^{-1} \int_{\mathrm{St}_{O(V)}(x)\left(k_{v}\right) \backslash O(V)\left(k_{v}\right)} \omega_{v}\left(g_{v}\right) \varphi_{v}\left(h_{v}^{-1} x\right) \tilde{d} h_{v}\right) .
\end{aligned}
$$

Lemma A.1. Suppose $\operatorname{dim}(V) \geq n$. For each $\beta_{v} \in \operatorname{Sym}_{n}\left(k_{v}\right)$ with $\operatorname{det} \beta_{v} \neq 0$, let

$$
\mathbb{T}_{\beta_{v}}:=\left\{\begin{array}{l|c}
T \in \operatorname{Hom}_{\mathbb{C}}\left(S\left(V\left(k_{v}\right)^{n}\right), \mathbb{C}\right) \mid \begin{array}{c}
T\left(\omega_{v}(\mathfrak{n}(b), h) \varphi_{v}\right)=\psi_{v}\left(\operatorname{Tr}\left(b \beta_{v}\right)\right) T\left(\varphi_{v}\right), \\
\forall b \in \operatorname{Sym}_{n}\left(k_{v}\right), h \in \mathrm{O}(V)\left(k_{v}\right), \varphi_{v} \in S\left(V\left(k_{v}\right)^{n}\right)
\end{array}
\end{array}\right\} .
$$

Then $\mathbb{T}_{\beta_{v}}=0$ if $\left(Q_{V}^{(n)}\right)^{-1}\left(\beta_{v}\right)\left(k_{v}\right)$ is empty. When $\left(Q_{V}^{(n)}\right)^{-1}\left(\beta_{v}\right)\left(k_{v}\right)$ is not empty, $\mathbb{T}_{\beta_{v}}$ is a one dimensional $\mathbb{C}$-vector space spanned by the following functional

$$
T_{\beta_{v}}: \varphi_{v} \longmapsto \int_{\left(Q_{V}^{(n)}\right)^{-1}\left(\beta_{v}\right)\left(k_{v}\right)} \varphi_{v} \delta_{\beta_{v}}=\int_{\mathrm{St}_{\mathrm{O}(V)}\left(x_{v}^{0}\right)\left(k_{v}\right) \backslash \mathrm{O}(V)\left(k_{v}\right)} \varphi_{v}\left(h_{v}^{-1} x_{v}^{0}\right) \tilde{d} h_{v}
$$

where $x_{v}^{0}$ is in $\left(Q_{V}^{(n)}\right)^{-1}\left(\beta_{v}\right)\left(k_{v}\right)$.

Proof. It is observed that the restriction map $S\left(V\left(k_{v}\right)^{n}\right) \rightarrow S\left(\left(Q_{V}^{(n)}\right)^{-1}\left(\beta_{v}\right)\right)$ induces an embedding from $\mathbb{T}_{\beta_{v}}$ into $\operatorname{Hom}_{\mathbb{C}}\left(S\left(\left(Q_{V}^{(n)}\right)^{-1}\left(\beta_{v}\right)\right), \mathbb{C}\right)$. Since every $\mathrm{O}(V)\left(k_{v}\right)$-invariant functional on $S\left(\left(Q_{V}^{(n)}\right)^{-1}\left(\beta_{v}\right)\right)$ must be a scalar multiple of $T_{\beta_{v}}$, the result holds. 
Remark A.2. Given $x \in V^{n}, Q_{V}^{(n)}$ is submersive at $x$ if $d Q_{V}^{(n)}(x): V^{n} \rightarrow \operatorname{Sym}_{n}$ is surjective. Take $\varphi_{v} \in S\left(V\left(k_{v}\right)^{n}\right)$ which is supported on the vectors in $V\left(k_{v}\right)^{n}$ where $d Q_{V}^{(n)}$ is submersive. Define the function $T_{\varphi_{v}} \in S\left(\operatorname{Sym}_{n}\left(k_{v}\right)\right)$ by

$$
T_{\varphi_{v}}\left(b_{v}\right)= \begin{cases}0, & \text { if }\left(Q_{V}^{(n)}\right)^{-1}\left(b_{v}\right)\left(k_{v}\right) \text { is empty, } \\ \int_{\left(Q_{V}^{(n)}\right)_{\operatorname{reg}}^{-1}\left(b_{v}\right)\left(k_{v}\right)} \varphi_{v} \delta_{b_{v}}, & \text { otherwise. }\end{cases}
$$

Then for every $\beta \in \operatorname{Sym}_{n}(k)$ with $\operatorname{det} \beta \neq 0$ and $a_{v} \in \mathrm{GL}_{n}\left(k_{v}\right)$,

$$
\begin{aligned}
& \int_{\operatorname{Sym}_{n}\left(k_{v}\right)} \Phi_{\varphi_{v}}\left(w_{n} \mathfrak{n}\left(b_{v}\right) \mathfrak{m}\left(a_{v}\right), s_{(n)}\right) \psi_{v}\left(-\operatorname{Tr}\left(b_{v} \beta\right)\right) d b_{v} \\
= & \int_{\operatorname{Sym}_{n}\left(k_{v}\right)} T_{\varphi_{v}}^{\wedge}\left(b_{v}\right) \psi_{v}\left(-\operatorname{Tr}\left(b_{v} \cdot{ }^{t} a_{v} \beta a_{v}\right)\right) d b_{v} \\
= & T_{\varphi_{v}}\left({ }^{t} a_{v} \beta a_{v}\right) .
\end{aligned}
$$

Here $T_{\varphi_{v}}^{\wedge}\left(b_{v}\right)$ is the Fourier transform of $T_{\varphi_{v}}$, i.e.

$$
T_{\varphi_{v}}^{\wedge}\left(b_{v}\right)=\int_{\operatorname{Sym}_{n}\left(k_{v}\right)} T_{\varphi_{v}}\left(b_{v}^{\prime}\right) \psi_{v}\left(\operatorname{Tr}\left(b_{v} b_{v}^{\prime}\right)\right) d b_{v}^{\prime} .
$$

On the other hand, we have

Lemma A.3. For $g \in \operatorname{Sp}_{n}\left(\mathbb{A}_{k}\right), \Phi \in I_{\mathbb{A}_{k}}(s)$, and $\beta=\left(\begin{array}{cc}0 & 0 \\ 0 & \beta_{0}\end{array}\right)$ with $\beta_{0} \in \operatorname{Sym}_{n-r}\left(k_{v}\right)$, $0 \leq r<n$, and $\operatorname{det} \beta_{0} \neq 0$,

$$
=\sum_{i \leq r} \sum_{a^{\prime} \in Q_{r-i}^{(r)}(k) \backslash \mathrm{GL}_{r}(k)} \int_{\operatorname{Sym}_{n-i}\left(\mathbb{A}_{k}\right)} \Phi\left(w_{n-i} \mathfrak{n}\left(b^{\prime}\right) \gamma\left(a^{\prime}\right) g, s\right) \psi\left(-\operatorname{Tr}\left(b^{\prime} \beta_{0}\right)\right) d b^{\prime} .
$$

Here $\gamma\left(a^{\prime}\right)=\mathfrak{m}\left(\begin{array}{cc}a^{\prime} & 0 \\ 0 & I_{n-r}\end{array}\right)$ and we embed $\operatorname{Sym}_{n-i}$ into $\operatorname{Sym}_{n}$ by sending $b^{\prime}$ to $\left(\begin{array}{cc}0 & 0 \\ 0 & b^{\prime}\end{array}\right)$.

Proof. The argument is similar to Lemma 3.1. Therefore we omit the proof.

Now, we arrive at the main result of this section.

Theorem A.4. There exists a non-zero constant $c$ such that the function

$$
\left(I^{n}\right)^{\prime}(g, \varphi):=I(g, \varphi)-c E\left(g, s_{(n)}, \Phi_{\varphi}\right)
$$

satisfies $\left(I^{n}\right)_{\beta}^{\prime}(g, \varphi)=0$ for every $g \in \operatorname{Sp}_{n}\left(\mathbb{A}_{k}\right), \varphi \in S\left(V\left(\mathbb{A}_{k}\right)^{n}\right)$, and $\beta \in \operatorname{Sym}_{n}(k)$ with $\operatorname{det} \beta \neq 0$.

Proof. Without loss of generality, assume $g=\mathfrak{m}(a)$ for $a \in \mathrm{GL}_{n}\left(\mathbb{A}_{k}\right)$ and $\varphi=\otimes_{v} \varphi_{v}$ is a pure tensor. It is clear that the functional

$$
T_{a}^{\prime}:=\left(\varphi \longmapsto E_{\beta}\left(\mathfrak{m}(a), s_{(n)}, \Phi_{\varphi}\right), \quad \forall \varphi \in S\left(V\left(\mathbb{A}_{k}\right)^{n}\right)\right)
$$

satisfies that

$$
T_{a}^{\prime}(\omega(\mathfrak{n}(b)) \varphi)=\psi\left(\operatorname{Tr}\left(b \cdot{ }^{t} a \beta a\right)\right) T_{a}^{\prime}(\varphi), \quad \forall b \in \operatorname{Sym}_{n}\left(\mathbb{A}_{k}\right) .
$$

Therefore Lemma A.1 implies that

$$
E_{\beta}\left(\mathfrak{m}(a), s_{(n)}, \Phi_{\varphi}\right)=I_{\beta}^{n}(\mathfrak{m}(a), \varphi)=0
$$

if $\left(Q_{V}^{(n)}\right)^{-1}(\beta)(k)$ is empty.

When $\left(Q_{V}^{(n)}\right)^{-1}(\beta)(k)$ is not empty, $\operatorname{dim}(V) \geq n$ and by Lemma A.1 again we can find a constant $c=c(\beta, a) \in \mathbb{C}$ such that

$$
E_{\beta}\left(\mathfrak{m}(a), s_{(n)}, \Phi_{\varphi}\right)=c(\beta, a) \cdot I_{\beta}^{n}(\mathfrak{m}(a), \varphi) .
$$


It remains to show that $c$ is non-zero and is independent of the choices of $\beta$ and $a$.

Lemma A.3 says that

$$
E_{\beta}\left(\mathfrak{m}(a), s_{(n)}, \Phi_{\varphi}\right)=\prod_{v}\left(\int_{\operatorname{Sym}_{n}\left(k_{v}\right)} \Phi_{\varphi}\left(w_{n} \mathfrak{n}\left(b_{v}\right) \mathfrak{m}\left(a_{v}\right), s_{(n)}\right) \psi_{v}\left(-\operatorname{Tr}\left(b_{v} \beta\right)\right) d b_{v}\right) .
$$

Let $\Sigma\left(a, Q_{V}, d b\right)$ be the finite set of places of $k$ such that when $v \notin \Sigma\left(a, Q_{V}, d b\right)$, we have $a_{v} \in \mathrm{GL}_{n}\left(O_{v}\right), \beta_{v} \in \operatorname{Sym}_{n}\left(O_{v}\right) \cap \mathrm{GL}_{n}\left(O_{v}\right), Q_{V}$ is unramified at $v$ and $\operatorname{vol}\left(\operatorname{Sym}_{n}\left(O_{v}\right), d b_{v}\right)=1$. For $v \notin \Sigma\left(a, Q_{V}, d b\right)$, take $\varphi_{v}^{0} \in S\left(V\left(k_{v}\right)^{n}\right)$ such that $\Phi_{\varphi_{v}^{0}}=\Phi_{v}^{0}$ (recall that $\Phi_{v}^{0}\left(\kappa_{v}, s\right)=1$ for all $\left.\kappa_{v} \in \operatorname{Sp}_{n}\left(O_{v}\right)\right)$. Then we get

$$
\begin{gathered}
\int_{\operatorname{Sym}_{n}\left(k_{v}\right)} \Phi_{\varphi_{v}^{0}}\left(w_{n} \mathfrak{n}\left(b_{v}\right) \mathfrak{m}\left(a_{v}\right), s\right) \psi_{v}\left(-\operatorname{Tr}\left(b_{v} \beta\right)\right) d b_{v} \\
=L_{v}\left(s+\frac{n+1}{2}, \chi_{V, v}\right)^{-1} \prod_{i=1}^{\left\lfloor\frac{n}{2}\right\rfloor} \zeta_{v}(2 s+n+1-2 i)^{-1} \\
\\
\cdot \begin{cases}L_{v}\left(s+1 / 2, \chi_{V, v} \chi_{\beta, n, v}\right), & \text { if } n \text { is even, } \\
1, & \text { if } n \text { is odd. }\end{cases}
\end{gathered}
$$

Here $\chi_{\beta, n, v}: k_{v}^{\times} \rightarrow\{ \pm 1\}$ is defined by

$$
\chi_{\beta, n, v}\left(\alpha_{v}\right):=\left((-1)^{n / 2} \operatorname{det} \beta, \alpha_{v}\right)_{v}, \quad \forall \alpha_{v} \in k_{v}^{\times} .
$$

Given $a_{1}, a_{2} \in \mathrm{GL}_{n}\left(\mathbb{A}_{k}\right)$, we choose $\varphi_{v}^{1}, \varphi_{v}^{2} \in S\left(V\left(k_{v}\right)^{n}\right)$ for every place $v$ as follows. Let $\Sigma:=\Sigma\left(a_{1}, Q_{V}, d b\right) \cup \Sigma\left(a_{2}, Q_{V}, d b\right)$. For $v \notin \Sigma$ we let $\varphi_{v}^{1}=\varphi_{v}^{2}=\varphi_{v}^{0}$; and for $v \in \Sigma$ we choose $\varphi_{v}^{i}$ which are supported on the vectors in $V\left(k_{v}\right)^{n}$ where $d Q_{V}^{(n)}$ is submersive and $T_{\varphi_{v}^{i}}\left({ }^{t} a_{v, i} \beta a_{v, i}\right) \neq 0$. Define

$$
\begin{aligned}
\Lambda_{\Sigma}(s):= & L_{\Sigma}\left(s+\frac{n+1}{2}, \chi_{V}\right)^{-1} \prod_{i=1}^{\left\lfloor\frac{n}{2}\right\rfloor} \zeta_{\Sigma}(2 s+n+1-2 i)^{-1} \\
& \cdot \begin{cases}L_{\Sigma}\left(s+1 / 2, \chi_{V} \chi_{\beta, n}\right), & \text { if } n \text { is even, } \\
1, & \text { if } n \text { is odd } .\end{cases}
\end{aligned}
$$

Then the discussion in Remark A.2 implies that

$$
\Lambda_{\Sigma}\left(s_{(n)}\right)=c\left(\beta, a_{i}\right)\left(\prod_{v \in \Sigma} \frac{1}{\lambda_{v}(\beta)}\right) \operatorname{vol}\left(\operatorname{St}_{\mathrm{O}(V)}(x)(k) \backslash \operatorname{St}_{\mathrm{O}(V)}\left(\mathbb{A}_{k}\right), d h^{\prime}\right) \prod_{v \notin \Sigma} \frac{T_{\beta_{v}}\left(\varphi_{v}^{0}\right)}{\lambda_{v}(\beta)},
$$

where $\left\{\lambda_{v}(\beta)\right\}$ is a convenient set of convergent factors for the gauge on $\left(Q_{V}^{(n)}\right)^{-1}(\beta)$ and $x$ is a chosen element in $\left(Q_{V}^{(n)}\right)^{-1}(\beta)$. Hence $c$ is independent of the choice of $a$. Finally, let $\left\{\lambda_{v}^{\prime}\right\}$ be a set of convergence factors for the measure $d h$ on $\mathrm{O}(V)\left(\mathbb{A}_{k}\right)$, then $\left\{\lambda_{v}^{\prime \prime}:=\lambda_{v}^{\prime} / \lambda_{v}(\beta)\right\}$ is a set of convergence factors for the measure $d h^{\prime}$ on $\mathrm{St}_{\mathrm{O}(V)}(x)\left(\mathbb{A}_{k}\right)$. Choosing suitable convergence factors $\left\{\lambda_{v}^{\prime \prime}\right\}$ for the measure $d h^{\prime}$ on $\mathrm{St}_{\mathrm{O}(V)}(x)\left(\mathbb{A}_{k}\right)$, it can be shown that $c$ is also independent of the choice of $\beta$. Therefore the proof is complete.

One consequence of Theorem A.4 is:

Theorem A.5. Given a Schwartz function $\varphi \in S\left(V\left(\mathbb{A}_{k}\right)^{n}\right)$, we have that for every cusp form $f$ on $\operatorname{Sp}_{n}\left(\mathbb{A}_{k}\right)$,

$$
\int_{\operatorname{Sp}_{n}(k) \backslash \operatorname{Sp}_{n}\left(\mathbb{A}_{k}\right)} I^{n}(g, \varphi) f(g) d g=0 .
$$


Proof. The argument is similar to Theorem 2.7 in [9]. We recall the key steps for the sake of completeness.

Consider the auxiliary Eisenstein series on $\operatorname{Sp}_{n}\left(\mathbb{A}_{k}\right)$ :

$$
\mathcal{E}^{0}(g, s):=\sum_{\gamma \in P_{n}(k) \backslash \operatorname{Sp}_{n}(k)} \Phi^{0}(\gamma g, s)
$$

where for $g=\left(\begin{array}{cc}a & * \\ 0 & { }^{t} a^{-1}\end{array}\right) \kappa$ with $a \in \mathrm{GL}_{n}\left(\mathbb{A}_{k}\right)$ and $\kappa \in \operatorname{Sp}_{n}\left(O_{\mathbb{A}_{k}}\right)$,

$$
\Phi^{0}(g, s):=|\operatorname{det} a|_{\mathbb{A}_{k}}^{s+(n+1) / 2} .
$$

It is known that $\mathcal{E}^{0}(g, s)$ has meromorphic continuation to the whole $s$-plane, and has a simple pole at $s=(n+1) / 2$ with residue $c_{1}$ independent of $g$. Then for any cusp form $f$ on $\operatorname{Sp}_{n}\left(\mathbb{A}_{k}\right)$,

$$
\operatorname{Res}_{s=(n+1) / 2}\left\langle\left(I^{n}\right)^{\prime}(\varphi), \mathcal{E}^{0}(s) f\right\rangle=c_{1}\left\langle\left(I^{n}\right)^{\prime}(\varphi), f\right\rangle=c_{1}\left\langle I^{n}(\varphi), f\right\rangle .
$$

Here

$$
\left\langle f_{1}, f_{2}\right\rangle:=\int_{\operatorname{Sp}_{n}(k) \backslash \operatorname{Sp}_{n}\left(\mathbb{A}_{k}\right)} f_{1}(g) f_{2}(g) d g .
$$

The second equality in (A.1) holds by Proposition 3.2. It suffices to show that

$$
\left\langle\left(I^{n}\right)^{\prime}(\varphi), \mathcal{E}^{0}(s) f\right\rangle=0 .
$$

It is observed that

$$
=\sum_{\beta \bmod \mathrm{GL}_{n}(k)} \int_{\left.M_{\beta}\left(\mathbb{A}_{k}\right) N_{n}\left(I^{n}\right)^{\prime}\right) \backslash \mathrm{Sp}_{n}\left(\mathbb{A}_{k}\right)} \int_{M_{\beta}(k) \backslash M_{\beta}\left(\mathbb{A}_{k}\right)}\left(I^{n}\right)_{\beta}^{\prime}(m g) f_{-\beta}(m g) \Phi^{0}(m g, s) d m d g .
$$

The sum runs over representatives $\beta \bmod \mathrm{GL}_{n}(k)$ in $\operatorname{Sym}_{n}(k)$. The action of $\mathrm{GL}_{n}(k)$ on $\operatorname{Sym}_{n}(k)$ is defined by $a * \beta:={ }^{t} a \beta a$, and

$$
M_{\beta}:=\left\{\mathfrak{m}(a) \in M_{n}: a \in \mathrm{GL}_{n} \text { such that } a * \beta=\beta\right\} .
$$

Theorem A.5 tells us that the sum runs over singular $\beta$. For convention, we choose $\beta$ to be of the form $\left(\begin{array}{cc}0 & 0 \\ 0 & \beta_{0}\end{array}\right)$ where $\beta_{0} \in \operatorname{Sym}_{n-r}(k)$ with $0<r \leq n$. Note that $M_{\beta}=L_{\beta} \cdot U_{\beta}$ where

$$
U_{\beta} \cong\left\{u(x)=\left(\begin{array}{cc}
I_{r} & x \\
0 & I_{n-r}
\end{array}\right) \mid x \in \operatorname{Mat}_{r \times(n-r)}\right\}
$$

and

Thus

$$
L_{\beta} \cong\left\{\left(\begin{array}{ll}
a & 0 \\
0 & d
\end{array}\right) \mid a \in \mathrm{GL}_{r}, d \in \mathrm{GL}_{n-r} \text { with }{ }^{t} d \beta_{0} d=\beta_{0}\right\}
$$

$$
\begin{aligned}
& \int_{M_{\beta}(k) \backslash M_{\beta}\left(\mathbb{A}_{k}\right)}\left(I^{n}\right)_{\beta}^{\prime}(m g) f_{-\beta}(m g) \Phi^{0}(m g, s) d m \\
= & \int_{L_{\beta}(k) \backslash L_{\beta}\left(\mathbb{A}_{k}\right)}\left(\int_{U_{\beta}(k) \backslash U_{\beta}\left(\mathbb{A}_{k}\right)}\left(I^{n}\right)_{\beta}^{\prime}(u l g) f_{-\beta}(u l g) d u\right) \Phi^{0}(l g, s) d l .
\end{aligned}
$$

For $\alpha \in \operatorname{Mat}_{r \times(n-r)}(k)$, denote

$$
\left(I^{n}\right)_{\alpha, \beta}^{\prime}(g):=\int_{\operatorname{Mat}_{r \times(n-r)}(k) \backslash \operatorname{Mat}_{r \times(n-r)}\left(\mathbb{A}_{k}\right)}\left(I^{n}\right)_{\beta}^{\prime}(u(x) g) \psi\left(-\operatorname{Tr}\left({ }^{t} \alpha x\right)\right) d x .
$$


Then

$$
\int_{U_{\beta}(k) \backslash U_{\beta}\left(\mathbb{A}_{k}\right)}\left(I^{n}\right)_{\beta}^{\prime}(u l g) f_{-\beta}(u l g) d u=\sum_{\alpha \in \mathrm{Mat}_{r \times(n-r)}(k)}\left(I^{n}\right)_{\alpha, \beta}^{\prime}(l g) f_{-\alpha,-\beta}(l g) .
$$

By straightforward calculation, we obtain:

(i) If $\beta=0$, then $f_{\beta}=0$.

(ii) If singular $\beta \neq 0$, then $f_{0, \beta}=0$.

(iii) If $\beta$ is singular and $\alpha \neq 0$, then $E_{\alpha, \beta}\left(\cdot, s, \Phi_{\varphi}\right)=0$.

(iv) If $\beta$ is singular and $\alpha \neq 0$, then $I_{\alpha, \beta}^{n}(\cdot, \varphi)=0$.

We point out that (iii) is deduced from the expression of $E_{\beta}\left(\cdot, s, \Phi_{\varphi}\right)$ in Lemma A.3. These observation completes the proof.

\section{Appendix B. On the Jacquet module of $S\left(V\left(k_{v}\right)^{n}\right)$ With Respect to $P_{n}$}

In this section, we describe the Jordan structure of the Jacquet module of $S\left(V\left(k_{v}\right)^{n}\right)$ (which is studied in [8] for the number field case). Recall that $\left(V, Q_{V}\right)$ is an anisotropic quadratic space over $k$, and $\omega_{v}$ is the Weil representation of $\operatorname{Sp}_{n}\left(k_{v}\right) \times \mathrm{O}(V)\left(k_{v}\right)$ on the Schwartz space $S\left(V\left(k_{v}\right)^{n}\right)$ for each place $v$ of $k$. The Jacquet module $\mathcal{J}_{n}$ of $S\left(V\left(k_{v}\right)^{n}\right)$ with respect to $P_{n}$ is the quotient space

$$
\frac{S\left(V\left(k_{v}\right)^{n}\right)}{\operatorname{Span}\left\{\omega_{v}(\mathfrak{n}(b)) \varphi-\varphi \mid b \in \operatorname{Sym}_{n}\left(k_{v}\right), \varphi \in S\left(V\left(k_{v}\right)^{n}\right)\right\}} .
$$

We modify the action of $\mathrm{GL}_{n}\left(k_{v}\right)$ on $\mathcal{J}_{n}$ by:

$$
\tilde{\omega}_{v}(a) \bar{\varphi}:=|\operatorname{det} a|_{v}^{-\frac{n+1}{2}} \cdot \overline{\omega_{v}(\mathfrak{m}(a)) \varphi}, \quad \forall a \in \mathrm{GL}_{n}\left(k_{v}\right) \text { and } \bar{\varphi} \in \mathcal{J}_{n} .
$$

Define $V\left(k_{v}\right)_{0}^{n}:=\left\{x \in V\left(k_{v}\right)^{n} \mid Q_{V}^{(n)}(x)=0\right\}$, where $Q_{V}^{(n)}: V^{n} \rightarrow \operatorname{Sym}_{n}$ is the moment map introduced in Section 1.3. The Schwartz space $S\left(V\left(k_{v}\right)_{0}^{n}\right)$ is invariant under the action of $\mathrm{GL}_{n}\left(k_{v}\right) \times \mathrm{O}(V)\left(k_{v}\right)$ defined by

$$
(a, h) \cdot \varphi(x)=\chi_{V, v}(\operatorname{det} a)|\operatorname{det} a|_{v}^{\frac{\operatorname{dim}(V)-(n+1)}{2}} \varphi\left(h^{-1} x a\right) .
$$

Let $l_{0}:=\min (l, n)$ where $l$ is the dimension of a maximal isotropic subspace of $V\left(k_{v}\right)$ (which is 0 or $\operatorname{dim}(V) / 2)$. It is clear that every $x=\left(x_{1}, \ldots, x_{n}\right) \in V\left(k_{v}\right)_{0}^{n}$ satisfies $\operatorname{dim}(\operatorname{Span} x) \leq l_{0}$, where Span $x:=\operatorname{Span}\left\{x_{1}, \ldots, x_{n}\right\}$. Thus

$$
V\left(k_{v}\right)_{0}^{n}=\coprod_{i=0}^{l_{0}} V\left(k_{v}\right)_{0, i}^{n}
$$

where

$$
V\left(k_{v}\right)_{0, i}^{n}:=\left\{x \in V\left(k_{v}\right)_{0}^{n} \mid \operatorname{dim}(\operatorname{Span} x)=i\right\} .
$$

Proposition B.1. (1) As a $\mathrm{GL}_{n}\left(k_{v}\right) \times \mathrm{O}(V)\left(k_{v}\right)$-module, $\mathcal{J}_{n}$ is isomorphic to $S\left(V\left(k_{v}\right)_{0}^{n}\right)$, where the isomorphism is induced by the restriction from $S\left(V\left(k_{v}\right)^{n}\right)$ to $S\left(V\left(k_{v}\right)_{0}^{n}\right)$.

(2) We have a $\mathrm{GL}_{n}\left(k_{v}\right) \times \mathrm{O}(V)\left(k_{v}\right)$-invariant filtration

$$
\mathcal{J}_{n}=\mathcal{J}_{n}^{(0)} \supset \mathcal{J}_{n}^{(1)} \supset \cdots \supset \mathcal{J}_{n}^{\left(l_{0}\right)} \supset \mathcal{J}_{n}^{\left(l_{0}+1\right)}=\{0\}
$$

such that as $\mathrm{GL}_{n}\left(k_{v}\right) \times \mathrm{O}(V)\left(k_{v}\right)$-modules,

$$
\widetilde{\mathcal{J}}_{n}^{(i)}:=\mathcal{J}_{n}^{(i)} / \mathcal{J}_{n}^{(i+1)} \cong S\left(V\left(k_{v}\right)_{0, i}^{n}\right) .
$$


Proof. Consider the following exact sequence:

$$
0 \rightarrow S\left(V\left(k_{v}\right)^{n}-V\left(k_{v}\right)_{0}^{n}\right) \rightarrow S\left(V\left(k_{v}\right)^{n}\right) \rightarrow S\left(V\left(k_{v}\right)_{0}^{n}\right) \rightarrow 0 .
$$

It is clear that $\omega_{v}(\mathfrak{n}(b)) \varphi-\varphi \in S\left(V\left(k_{v}\right)^{n}-V\left(k_{v}\right)_{0}^{n}\right)$ for every $b \in \operatorname{Sym}_{n}\left(k_{v}\right)$ and $\varphi \in$ $S\left(V\left(k_{v}\right)^{n}\right)$. On the other hand, for $x \in V\left(k_{v}\right)^{n}-V\left(k_{v}\right)_{0}^{n}$, we can find $b \in \operatorname{Sym}_{n}\left(k_{v}\right)$ such that $\psi_{v}\left(\operatorname{Trace}\left(b Q_{V}^{(n)}(x)\right)\right) \neq 1$. Choose a sufficiently small neighborhood $U_{x}$ of $x$ in $V\left(k_{v}\right)^{n}-$ $V\left(k_{v}\right)_{0}^{n}$ such that

$$
\psi_{v}\left(\operatorname{Trace}\left(b Q_{V}^{(n)}\left(x^{\prime}\right)\right)\right)=\psi_{v}\left(\operatorname{Trace}\left(b Q_{V}^{(n)}(x)\right)\right), \quad \forall x^{\prime} \in U_{x} .
$$

Let $\varphi_{x}$ be the characteristic function of $U_{x}$. Then

$$
\omega_{v}(\mathfrak{n}(b)) \varphi_{x}-\varphi_{x}=\left(\psi_{v}\left(\operatorname{Trace}\left(b Q_{V}^{(n)}(x)\right)\right)-1\right) \varphi_{x} .
$$

This implies that the Schwartz space $S\left(V\left(k_{v}\right)^{n}-V\left(k_{v}\right)_{0}^{n}\right)$ coincides with

$$
\operatorname{Span}\left\{\omega_{v}(\mathfrak{n}(b)) \varphi-\varphi \mid b \in \operatorname{Sym}_{n}\left(k_{v}\right), \varphi \in S\left(V\left(k_{v}\right)^{n}\right)\right\} .
$$

Therefore the proof of (1) is complete.

Identifying $\mathcal{J}_{n}=\mathcal{J}_{n}^{(0)}$ with $S\left(V\left(k_{v}\right)_{0}^{n}\right)$, for $0<i \leq l_{0}$ we let

$$
\mathcal{J}_{n}^{(i)}:=\left\{\varphi \in S\left(V\left(k_{v}\right)_{0}^{n}\right) \mid \varphi(x)=0, \forall x \in \coprod_{j=0}^{i-1} V\left(k_{v}\right)_{0, j}^{n},\right\} .
$$

Then for $0 \leq i \leq l_{0}$,

$$
\mathcal{J}_{n} / \mathcal{J}_{n}^{(i+1)} \cong S\left(\coprod_{j=0}^{i} V\left(k_{v}\right)_{0, j}^{n}\right)
$$

This assures that for $0 \leq i \leq l_{0}$,

$$
\mathcal{J}_{n}^{(i)} / \mathcal{J}_{n}^{(i+1)} \cong S\left(V\left(k_{v}\right)_{0, i}^{n}\right)
$$

and completes the proof of (2).

Choose $x_{1}, \ldots, x_{l} \in V\left(k_{v}\right)$ such that $\operatorname{Span}\left\{x_{1}, \ldots, x_{l}\right\}$ is a maximal isotropic subspace. Then there exist $x_{1}^{\prime}, \ldots, x_{l}^{\prime} \in V\left(k_{v}\right)$ such that

$$
\begin{gathered}
<x_{i}^{\prime}, x_{j}^{\prime}>_{V}=0, \quad 1 \leq i, j \leq l, \\
<x_{i}, x_{j}^{\prime}>_{V}=0, \quad i \neq j, \quad \text { and }<x_{i}, x_{i}^{\prime}>_{V}=1 .
\end{gathered}
$$

For $0 \leq i \leq l$, let $V\left(k_{v}\right)^{(i)}$ be the orthogonal complement of $\operatorname{Span}\left\{x_{1}, \ldots, x_{i}, x_{1}^{\prime}, \ldots, x_{i}^{\prime}\right\}$ in $V\left(k_{v}\right)$. Define

$$
P_{i}^{\prime}:=\left\{h \in \mathrm{O}(V)\left(k_{v}\right) \mid h \cdot \operatorname{Span}\left\{x_{1}, \ldots, x_{i}\right\}=\operatorname{Span}\left\{x_{1}, \ldots, x_{i}\right\}\right\},
$$

which is a parabolic subgroup of $\mathrm{O}(V)\left(k_{v}\right)$ whose Levi subgroup $M_{i}^{\prime}$ is isomorphic to $\mathrm{GL}_{i}\left(k_{v}\right) \times$ $\mathrm{O}\left(V\left(k_{v}\right)^{(i)}\right)$. More precisely, let $\left\{x_{1}^{\prime}, \ldots, x_{l}^{\prime}, x_{1}^{\prime \prime}, \ldots, x_{\operatorname{dim}(V)-2 l}^{\prime \prime}, x_{1}, \ldots, x_{l}\right\}$ be a basis of $V$. Then with respect to this basis, elements in $M_{i}^{\prime}$ are of the form

$$
(a, b)=\left(\begin{array}{ccc}
{ }^{t} a^{-1} & 0 & 0 \\
0 & b & 0 \\
0 & 0 & a
\end{array}\right)
$$

where $a \in \mathrm{GL}_{i}\left(k_{v}\right)$ and $b \in \mathrm{O}\left(V\left(k_{v}\right)^{(i)}\right)$.

Take $0 \leq i \leq l_{0}$. Recall

$$
Q_{i}=\left\{\left(\begin{array}{cc}
a_{1} & * \\
0 & a_{2}
\end{array}\right) \in \mathrm{GL}_{n} \mid a_{1} \in \mathrm{GL}_{n-i}, a_{2} \in \mathrm{GL}_{i}\right\} .
$$


Define an action $\rho_{i}$ of $Q_{i}\left(k_{v}\right) \times P_{i}^{\prime}$ on $S\left(\mathrm{GL}_{i}\left(k_{v}\right)\right)$ by

$$
\rho_{i}\left(\left(\left(\begin{array}{cc}
a_{1} & * \\
0 & a_{2}
\end{array}\right),\left(a_{2}^{\prime}, h^{\prime}\right) \cdot n^{\prime}\right) \varphi\right)(g)=\varphi\left(a_{2}^{\prime-1} g a_{2}\right),
$$

where $\left(a_{2}^{\prime}, h^{\prime}\right) \in M_{i}^{\prime}$ and $n^{\prime}$ is in the unipotent radical of $P_{i}^{\prime}$. Let $\operatorname{Ind}\left(S\left(\mathrm{GL}_{i}\left(k_{v}\right)\right)\right)$ be the space of smooth functions $f$ from $\mathrm{GL}_{n}\left(k_{v}\right) \times \mathrm{O}(V)\left(k_{v}\right)$ to $S\left(\mathrm{GL}_{i}\left(k_{v}\right)\right)$ satisfying that for every element $(g, h) \in \mathrm{GL}_{n}\left(k_{v}\right) \times \mathrm{O}(V)\left(k_{v}\right)$,

$$
f\left(\left(b, b^{\prime}\right)(g, h)\right)=\mu_{i}(b) \cdot \rho_{i}\left(b, b^{\prime}\right)(f(g, h)), \quad \forall\left(b, b^{\prime}\right) \in Q_{i} \times P_{i}^{\prime},
$$

where for $b=\left(\begin{array}{cc}a_{1} & * \\ 0 & a_{2}\end{array}\right) \in Q_{i}\left(k_{v}\right)$,

$$
\mu_{i}(b)=\chi_{V, v}\left(\operatorname{det} a_{1} \operatorname{det} a_{2}\right)\left|\operatorname{det} a_{1} \operatorname{det} a_{2}\right| \frac{\operatorname{dim}(V)-(n+1)}{v^{2}} .
$$

The action of $\mathrm{GL}_{n}\left(k_{v}\right) \times \mathrm{O}(V)\left(k_{v}\right)$ on $\operatorname{Ind}\left(S\left(\mathrm{GL}_{i}\left(k_{v}\right)\right)\right)$ is defined by right translation.

Proposition B.2. For $0 \leq i \leq l_{0}$, we have

$$
\widetilde{\mathcal{J}}_{n}^{(i)} \cong S\left(V\left(k_{v}\right)_{0, i}^{n}\right) \cong \operatorname{Ind}\left(S\left(\mathrm{GL}_{i}\left(k_{v}\right)\right)\right)
$$

as $\mathrm{GL}_{n}\left(k_{v}\right) \times \mathrm{O}(V)\left(k_{v}\right)$ modules.

Proof. For $0 \leq i \leq l_{0}$, Set $x^{(i)}:=\left(0, \ldots, 0, x_{1}, x_{2}, \ldots, x_{i}\right) \in V\left(k_{v}\right)_{0, i}^{n}$. Let $\iota_{1}$ and $\iota_{2}$ be the embeddings from $\mathrm{GL}_{i}\left(k_{v}\right)$ into $P_{i}^{\prime} \subset \mathrm{O}(V)\left(k_{v}\right)$ and $Q_{i}\left(k_{v}\right) \subset \mathrm{GL}_{n}\left(k_{v}\right)$, respectively. Then we have

$$
\iota_{1}\left(g^{\prime}\right) x^{(i)}=x^{(i)} \iota_{2}\left(g^{\prime}\right), \quad \forall g^{\prime} \in \mathrm{GL}_{i}\left(k_{v}\right) .
$$

Define a map $F$ from $S\left(V\left(k_{v}\right)_{0, i}^{n}\right)$ to $\operatorname{Ind}\left(S\left(\mathrm{GL}_{i}\left(k_{v}\right)\right)\right)$ by

$$
\varphi \longmapsto F_{\varphi}
$$

where for $(g, h) \in \mathrm{GL}_{n}\left(k_{v}\right) \times \mathrm{O}(V)\left(k_{v}\right)$,

$$
F_{\varphi}(g, h)=\left(g^{\prime} \mapsto \chi_{V, v}(\operatorname{det} g)|\operatorname{det} g|_{v}^{\frac{\operatorname{dim}(V)-(n+1)}{2}} \cdot \varphi\left(h^{-1} x^{(i)} \iota_{2}\left(g^{\prime}\right) g\right)\right) \in S\left(\mathrm{GL}_{i}\left(k_{v}\right)\right) .
$$

Then it is clear that $F$ is $\mathrm{GL}_{n}\left(k_{v}\right) \times \mathrm{O}(V)\left(k_{v}\right)$-equivariant. Since for every element $x \in$ $V\left(k_{v}\right)_{0, i}^{n}$ we can find $(g, h) \in \mathrm{GL}_{n}\left(k_{v}\right) \times \mathrm{O}(V)\left(k_{v}\right)$ such that $x=h^{-1} x^{(i)} g$, the inverse map $F^{-1}: \operatorname{Ind}\left(S\left(\mathrm{GL}_{i}\left(k_{v}\right)\right)\right) \rightarrow S\left(V\left(k_{v}\right)_{0, i}^{n}\right)$ can be defined by the following: for $f \in$ $\operatorname{Ind}\left(S\left(\mathrm{GL}_{i}\left(k_{v}\right)\right)\right)$,

$$
F_{f}^{-1}(x):=\chi_{V, v}(\operatorname{det} g)^{-1}|\operatorname{det} g|_{v}^{\frac{-\operatorname{dim}(V)+(n+1)}{2}} \cdot f(g, h)(1), \quad \forall x=h^{-1} x^{(i)} g \in V\left(k_{v}\right)_{0, i}^{n} .
$$

We remark that the modulus character $\delta_{i}$ of the parabolic subgroup $Q_{i}\left(k_{v}\right) \times P_{i}^{\prime}$ is:

$$
\delta_{i}\left(\left(\begin{array}{cc}
a_{1} & * \\
0 & a_{2}
\end{array}\right),\left(\begin{array}{ccc}
{ }^{t} a_{2}^{\prime-1} & * & * \\
0 & h^{\prime} & * \\
0 & 0 & a_{2}^{\prime}
\end{array}\right)\right)=\frac{\left|\operatorname{det} a_{1}\right|_{v}^{i}}{\left|\operatorname{det} a_{2}\right|_{v}^{n-i}} \cdot\left|\operatorname{det} a_{2}^{\prime}\right|_{v}^{-\operatorname{dim}(V)+i+1} .
$$

Recall that $s_{(n)}=\operatorname{dim}(V) / 2-(n+1) / 2$, and $I_{v}\left(-s_{(n)}\right)$ is the space of smooth functions $f$ on $\operatorname{Sp}_{n}\left(k_{v}\right)$ satisfying that

$$
f\left(\left(\begin{array}{cc}
a & * \\
0 & { }^{t} a^{-1}
\end{array}\right) g\right)=\chi_{V, v}(\operatorname{det} a)|\operatorname{det} a|_{v}^{-\frac{\operatorname{dim}(V)}{2}} f(g), \quad \forall a \in \mathrm{GL}_{n}\left(k_{v}\right), g \in \operatorname{Sp}_{n}\left(k_{v}\right) .
$$


In other words, $I_{v}\left(-s_{(n)}\right)$ is the Siegel-parabolic induction from the character $|\cdot|_{v}^{-s_{(n)}}$ on $P_{n}\left(k_{v}\right)$. Therefore the Frobenius reciprocity (cf. [1] Proposition 4.5.1) gives us

$$
\operatorname{Hom}_{\mathrm{Sp}_{n}\left(k_{v}\right) \times \mathrm{O}(V)\left(k_{v}\right)}\left(S\left(V\left(k_{v}\right)^{n}\right), I_{v}\left(-s_{(n)}\right) \otimes \mathbf{1}\right)=\operatorname{Hom}_{\mathrm{GL}_{n}\left(k_{v}\right) \times \mathrm{O}(V)\left(k_{v}\right)}\left(\mathcal{J}_{n},|\cdot|_{v}^{-s_{(n)}} \otimes \mathbf{1}\right) .
$$

Note that $\ell(n, v)=\operatorname{dim}\left(\operatorname{Hom}_{\mathrm{GL}_{n}\left(k_{v}\right) \times \mathrm{O}(V)\left(k_{v}\right)}\left(\mathcal{J}_{n},|\cdot|_{v}^{-s_{(n)}} \otimes \mathbf{1}\right)\right)$ is bounded by

$$
\sum_{i=0}^{l_{0}} \operatorname{dim}\left(\operatorname{Hom}_{\mathrm{GL}_{n}\left(k_{v}\right) \times \mathrm{O}(V)\left(k_{v}\right)}\left(\tilde{\mathcal{J}}_{n}^{(i)},\left.|\cdot|\right|_{v} ^{-s_{(n)}} \otimes \mathbf{1}\right)\right) .
$$

For each $i$, Proposition B.2 tells us that $\widetilde{\mathcal{J}}_{n}^{(i)}$ is also a parabolic induction. Hence by Frobenius reciprocity again, we get

$$
\operatorname{Hom}_{\mathrm{GL}_{n}\left(k_{v}\right) \times \mathrm{O}(V)\left(k_{v}\right)}\left(\widetilde{\mathcal{J}}_{n}^{(i)},\left.|\cdot|\right|_{v} ^{-s_{(n)}} \otimes \mathbf{1}\right)=\operatorname{Hom}_{R_{i}}\left(\left(\mu_{i} \delta_{i}^{-1}\right) \otimes \rho_{i},|\cdot| v^{-s_{(n)}} \otimes \mathbf{1}\right),
$$

where $R_{i}=\left(\mathrm{GL}_{n-i}\left(k_{v}\right) \times \mathrm{GL}_{i}\left(k_{v}\right)\right) \times\left(\mathrm{GL}_{i}\left(k_{v}\right) \times O\left(V\left(k_{v}\right)^{(i)}\right)\right)$ is the Levi subgroup of $Q_{i}\left(k_{v}\right) \times P_{i}^{\prime}$.

For $m=\left(a_{1}, a_{2}, a_{2}^{\prime}, h\right) \in R_{i}$,

$$
\mu_{i} \delta_{i}^{-1}(m)=\chi_{V, v}\left(\operatorname{det} a_{1} \operatorname{det} a_{2}\right)\left|\operatorname{det} a_{1}\right|_{v}^{s_{(n)}-i}\left|\operatorname{det} a_{2}\right|_{v}^{s_{(n)}+n-i} \cdot\left|\operatorname{det} a_{2}^{\prime}\right|_{v}^{\operatorname{dim}(V)-i-1} .
$$

Hence $\operatorname{Hom}_{R_{i}}\left(\left(\mu_{i} \delta_{i}^{-1}\right) \otimes \rho_{i},|\cdot|_{v}^{-s_{(n)}} \otimes \mathbf{1}\right)=0$ unless (i) $i=2 s_{(n)}=\operatorname{dim}(V)-(n+1)$ or (ii) $i=n$. In both cases we can get

$$
\operatorname{dim}\left(\operatorname{Hom}_{R_{i}}\left(\left(\mu_{i} \delta_{i}^{-1}\right) \otimes \rho_{i},|\cdot| v^{-s_{(n)}} \otimes \mathbf{1}\right)\right)=1 .
$$

Note that $i$ is bounded by $l_{0}=\min (l, n)$. We then arrive at

Lemma B.3. Let

$$
\ell(n, v):=\operatorname{dim}_{\mathbb{C}} \operatorname{Hom}_{\mathrm{Sp}_{n}\left(k_{v}\right) \times \mathrm{O}(V)\left(k_{v}\right)}\left(S\left(V\left(k_{v}\right)^{n}\right), I_{v}\left(-s_{(n)}\right) \otimes \mathbf{1}\right),
$$

where 1 is the trivial representation of $\mathrm{O}(V)\left(k_{v}\right)$. Then

(i) When $\operatorname{dim}(V)<n+1$, we have $\ell(n, v)=0$.

(ii) When $\operatorname{dim}(V)=2$ and $n=1$,

$$
\ell(n, v) \leq \begin{cases}1, & \text { if } V\left(k_{v}\right) \text { is anisotropic, } \\ 2, & \text { if } V\left(k_{v}\right) \text { is isotropic. }\end{cases}
$$

(iii) When $\operatorname{dim}(V)=4$ and $n=1$ or 2 ,

$$
\ell(n, v) \leq \begin{cases}n, & \text { if } V\left(k_{v}\right) \text { is isotropic, } \\ 0, & \text { if } V\left(k_{v}\right) \text { is anisotropic. }\end{cases}
$$

(iv) When $\operatorname{dim}(V)=4$ and $n=3$, we have $\ell(n, v) \leq 1$.

Remark B.4. When $\operatorname{dim}(V)=2, n=1$, and $V\left(k_{v}\right)$ is isotropic, the above discussion says that

$$
\ell(n, v)=\operatorname{dim}_{\mathbb{C}} \operatorname{Hom}_{\mathrm{GL}_{1}\left(k_{v}\right) \times \mathrm{GL}_{1}\left(k_{v}\right)}\left(\mathcal{J}_{1}\left(k_{v}\right), \mathbf{1}\right) .
$$

In this case, $\mathcal{J}_{1}\left(k_{v}\right) \cong S\left(k_{v}\right)$, and every homomorphism in $\operatorname{Hom}_{k_{v}^{\times} \times k_{v}^{\times}}\left(S\left(k_{v}\right), \mathbf{1}\right)$ must be of the form

$$
c \cdot(\varphi \longmapsto \varphi(0)), \quad c \in \mathbb{C} .
$$

Therefore $\ell(n, v)=1$ in this case, and the proof of Lemma 4.4 is complete. 


\section{Appendix C. Maass-Jacquet-Shalika Eisenstein series on $\mathrm{GL}_{n}\left(\mathbb{A}_{k}\right)$}

Fix an integer $r$ with $0<r<n$. Set $X_{r}:=\operatorname{Mat}_{r \times n}$ as an affine algebraic variety over $k$. Let $\mu_{1, v}, \mu_{2, v}$ be two characters on $k_{v}^{\times}$. For any $g \in \mathrm{GL}_{n}\left(k_{v}\right)$ and Schwartz function $f_{v} \in S\left(X_{r}\left(k_{v}\right)\right)$, define $F_{v}(g)=F_{v}\left(g, \mu_{1, v}, \mu_{2, v}, f_{v}\right)$ to be

$$
\mu_{1, v}(\operatorname{det} g)|\operatorname{det} g|_{v}^{r / 2} \int_{\mathrm{GL}_{r}\left(k_{v}\right)} f_{v}\left(h_{v}^{-1}\left(0, I_{r}\right) g\right) \mu_{v}^{-1}\left(\operatorname{det} h_{v}\right) d^{\times} h_{v} .
$$

Here $\mu_{v}=\mu_{1, v} \mu_{2, v}^{-1}|\cdot|{ }_{v}^{n / 2}$ and the Haar measure $d^{\times} h_{v}$ is normalized so that the volume of $\mathrm{GL}_{r}\left(O_{v}\right)$ is 1 for all $v$.

Lemma C.1. The integral $F_{v}(g)$ is absolutely convergent for every $g \in \mathrm{GL}_{n}\left(k_{v}\right)$ if $\left|\mu_{1, v} \mu_{2, v}^{-1}\right|=$ $|\cdot|_{v}^{\sigma}$ where $\sigma>r-n / 2-1$.

Proof. Without loss of generality, assume $f_{v}$ is the characteristic function of $X_{r}\left(O_{v}\right)$ and $g=1$. Then by straightforward computation we get

$$
\begin{aligned}
& \int_{\mathrm{GL}_{r}\left(k_{v}\right)}\left|f_{v}\left(h_{v}^{-1}\left(0, I_{r}\right)\right) \mu_{v}^{-1}\left(\operatorname{det} h_{v}\right)\right| d^{\times} h_{v} \\
= & \int_{\mathrm{GL}_{r}\left(k_{v}\right) \cap \operatorname{Mat}_{r}\left(O_{v}\right)}\left|\mu_{v}\right|^{-1} d^{\times} h_{v} \\
= & \prod_{i=0}^{r-1} \zeta_{v}\left(\sigma-\frac{n}{2}-i\right) .
\end{aligned}
$$

This assures the result.

Recall that

$$
Q_{r}=\left\{\left(\begin{array}{cc}
a_{1} & * \\
0 & a_{2}
\end{array}\right) \in \mathrm{GL}_{n} \mid a_{1} \in \mathrm{GL}_{n-r}, a_{2} \in \mathrm{GL}_{r}\right\} .
$$

For $g \in \mathrm{GL}_{n}\left(k_{v}\right)$ and $b=\left(\begin{array}{cc}a_{1} & * \\ 0 & a_{2}\end{array}\right) \in Q_{r}\left(k_{v}\right)$, it is clear that

$$
F_{v}(b g)=\mu_{1, v}\left(\operatorname{det} a_{1}\right) \mu_{2, v}\left(\operatorname{det} a_{2}\right)\left|\delta_{Q_{r}}(b)\right|_{v}^{1 / 2} F_{v}(g)
$$

where $\delta_{Q_{r}}(b)=\left(\operatorname{det} a_{1}\right)^{r} \cdot\left(\operatorname{det} a_{2}\right)^{r-n}$. Let $\tilde{I}_{v}\left(\mu_{1, v}, \mu_{2, v}\right)$ be the space of smooth functions $\Psi_{v}$ on $\mathrm{GL}_{n}\left(k_{v}\right)$ satisfying that for $g \in \mathrm{GL}_{n}\left(k_{v}\right)$ and $b=\left(\begin{array}{cc}a_{1} & * \\ 0 & a_{2}\end{array}\right) \in Q_{r}\left(k_{v}\right)$,

$$
\Psi_{v}(b g)=\mu_{1, v}\left(\operatorname{det} a_{1}\right) \mu_{2, v}\left(\operatorname{det} a_{2}\right)\left|\delta_{Q_{r}}(b)\right|_{v}^{1 / 2} \Psi_{v}(g) .
$$

Then the map $\left(f_{v} \mapsto F_{v}\right)$ gives us a $\mathrm{GL}_{n}\left(k_{v}\right)$-equivariant homomorphism from $S\left(X_{r}\left(k_{v}\right)\right)$ to $\tilde{I}_{v}\left(\mu_{1, v}, \mu_{2, v}\right)$.

Lemma C.2. (1) Given $\Psi_{v} \in \widetilde{I}_{v}\left(\mu_{1, v}, \mu_{2, v}\right)$, there exists a Schwartz function $f$ on $X_{r}\left(k_{v}\right)$ supported on elements of rank $r$ such that

$$
F_{v}\left(g, \mu_{1, v}, \mu_{2, v}, f_{v}\right)=\Psi_{v}(g), \quad \forall g \in \mathrm{GL}_{n}\left(k_{v}\right) .
$$

In other words, the map $\left(f_{v} \mapsto F_{v}\right)$ from $S\left(X_{r}\left(k_{v}\right)\right)$ to $\tilde{I}_{v}\left(\mu_{1, v}, \mu_{2, v}\right)$ is surjective.

(2) Suppose $\mu_{1, v}$ and $\mu_{2, v}$ are both unramified, i.e. $\mu_{1, v}\left(O_{v}\right)=\mu_{2, v}\left(O_{v}\right)=1$. Let $f_{v}^{0}$ be the charateristic function of $X_{r}\left(O_{v}\right)$. Then for $g \in \mathrm{GL}_{n}\left(k_{v}\right)$

$$
F_{v}^{0}(g):=F_{v}\left(g, \mu_{1, v}, \mu_{2, v}, f_{v}^{0}\right)=\prod_{i=0}^{r-1} \zeta_{v}\left(\sigma-\frac{n}{2}-r-i\right) \Psi_{v}^{0}(g)
$$

where $\mu_{1, v} \mu_{2, v}^{-1}=|\cdot|_{v}^{\sigma}$ and $\Psi_{v}^{0} \in \widetilde{I}_{v}\left(\mu_{1, v}, \mu_{2, v}\right)$ satisfies

$$
\Psi_{v}^{0}(\kappa)=1, \quad \forall \kappa \in \mathrm{GL}_{n}\left(O_{v}\right) .
$$


Proof. The Iwasawa decomposition allows us to write $g$ as $b \kappa$, where $b \in Q_{r}\left(k_{v}\right)$ and $\kappa \in$ $\mathrm{GL}_{n}\left(O_{v}\right)$. Hence we can assume $g=\kappa \in \mathrm{GL}_{n}\left(O_{V}\right)$. Then (2) follows from the proof of Lemma C.1. To prove (1), we take $f_{v} \in S\left(X_{r}\left(k_{v}\right)\right)$ such that the support of $f_{v}$ is contained in $\left(0, I_{r}\right) \cdot \mathrm{GL}_{n}\left(O_{v}\right)$ and

$$
f_{v}\left(\left(0, I_{r}\right) \kappa\right):=\mu_{1, v}(\operatorname{det} \kappa)^{-1} \psi_{v}(\kappa) .
$$

Then for each $\kappa \in \mathrm{GL}_{n}\left(O_{v}\right)$,

$$
\begin{aligned}
F_{v}(\kappa) & =\mu_{1, v}(\kappa) \int_{\mathrm{GL}_{r}\left(k_{v}\right)} f_{v}\left(h_{v}^{-1}\left(0, I_{r}\right) \kappa\right) \mu_{v}^{-1}\left(\operatorname{det} h_{v}\right) d^{\times} h_{v} \\
& =\mu_{1, v}(\kappa) \int_{\mathrm{GL}_{r}\left(O_{v}\right)} \mu_{1, v}\left(\operatorname{det} h_{v}^{-1} \operatorname{det} \kappa\right)^{-1} \Psi_{v}\left(\left(\begin{array}{cc}
I_{n-r} & 0 \\
0 & h_{v}^{-1}
\end{array}\right) \kappa\right) \mu_{v}^{-1}\left(\operatorname{det} h_{v}\right) d^{\times} h_{v} \\
& =\Psi_{v}(\kappa) .
\end{aligned}
$$

Therefore the proof is complete.

Let $\mu_{1}, \mu_{2}$ be two Hecke characters on $k^{\times} \backslash \mathbb{A}_{k}^{\times}$. For any $g \in \mathrm{GL}_{n}\left(\mathbb{A}_{k}\right)$ and Schwartz function $f \in S\left(X_{r}\left(\mathbb{A}_{k}\right)\right)$, we set

$$
F(g)=F\left(g, \mu_{1}, \mu_{2}, f\right):=\mu_{1}(\operatorname{det} g)|\operatorname{det} g|_{\mathbb{A}_{k}}^{r / 2} \int_{\mathrm{GL}_{r}\left(\mathbb{A}_{k}\right)} f\left(h^{-1}\left(0, I_{r}\right) g\right) \mu^{-1}(\operatorname{det} h) d^{\times} h .
$$

Here $\mu=\mu_{1} \mu_{2}^{-1}|\cdot|_{\mathbb{A}_{k}}^{n / 2}$ and the Haar measure $d^{\times} h=\prod_{v} d^{\times} h_{v}$. In particular, the volume of $\mathrm{GL}_{r}\left(O_{\mathbb{A}_{k}}\right)$ is 1. By Lemma C.1, this integral is absolutely convergent if $\left|\mu_{1} \mu_{2}^{-1}\right|=|\cdot|_{\mathbb{A}_{k}}^{\sigma}$ where $\sigma>r-n / 2$. The Maass-Jacquet-Shalika Eisenstein series associated to $f, \mu_{1}, \mu_{2}$ is defined by (cf. [7] and [13])

$$
E\left(g, \mu_{1}, \mu_{2}, f\right)=\sum_{\gamma \in Q_{r}(k) \backslash \mathrm{GL}_{n}(k)} F(\gamma g) .
$$

This series converges absolutely when $\left|\mu_{1} \mu_{2}^{-1}\right|_{\mathbb{A}_{k}}=|\cdot|_{\mathbb{A}_{k}}^{\sigma}$ with $\sigma>n / 2$ (cf. [14] II.1.5).

Theorem C.3. Suppose $\mu_{1} \cdot \mu_{2}^{-1}=|\cdot|_{\AA_{k}}^{\sigma}$. Then

(1) (Continuation) $E\left(g, \mu_{1}, \mu_{2}, f\right)$ can be extended to a meromorphic function in $\sigma$ (in fact, a rational function in $\left.q^{-\sigma}\right)$, and every possible pole can only be a simple pole. Let $P(\sigma):=P^{+}(\sigma) \cdot P^{-}(\sigma)$, where

$$
P^{ \pm}(\sigma)=\prod_{i=0}^{r-1}\left(1-q^{-\sigma \pm\left(\frac{n}{2}-i\right)}\right) .
$$

Then $P(\sigma) \cdot E\left(g, \mu_{1}, \mu_{2}, f\right)$ is entire.

(2) (Functional equation) For each $f \in S\left(X_{r}\left(\mathbb{A}_{k}\right)\right)$, we have

$$
E\left(g, \mu_{1}, \mu_{2}, f\right)=E\left({ }^{t} g^{-1}, \mu_{1}^{-1}, \mu_{2}^{-1}, f^{\wedge}\right)
$$

where $f^{\wedge}$ is the Fourier transform of $f$ :

$$
f^{\wedge}(x):=\int_{X_{r}\left(\mathbb{A}_{k}\right)} f(y) \psi\left(-\operatorname{Tr}\left(x^{t} y\right)\right) d y .
$$

The Haar measure $d y$ is chosen to be self-dual, i.e. $f^{\wedge \wedge}(x)=f(-x)$.

(3) Suppose that there exists a place $v$ of $k$ such that the support of the restriction of $f$ on $X_{r}\left(k_{v}\right)$ is contained in the set of elements with rank $r$ in $X_{r}\left(k_{v}\right)$. Then

$$
P^{+}(\sigma) \cdot E\left(g, \mu_{1}, \mu_{2}, f\right) \text { is entire. }
$$

Proof. Replacing $f$ to the Schwartz function $f(\cdot g)$, we can assume $g=1$ and set $E_{r}(\sigma, f):=$ $E\left(1, \mu_{1}, \mu_{2}, f\right)$. Let

$$
X_{r}^{(i)}(k):=\left\{x \in X_{r}(k): \operatorname{rank}(x)=i\right\}, \quad 0 \leq i \leq r .
$$


For $h \in \mathrm{GL}_{r}\left(\mathbb{A}_{k}\right), f \in S\left(X_{r}\left(\mathbb{A}_{k}\right)\right)$ we define

$$
\theta_{r}^{(i)}(h ; f):=\sum_{x \in X_{r}^{(i)}(k)} f\left(h^{-1} x\right)
$$

and

$$
\theta_{r}(h ; f):=\sum_{i=0}^{r} \theta_{r}^{(i)}(h ; f)=\sum_{x \in X_{r}(k)} f\left(h^{-1} x\right) .
$$

Then we have

$$
E_{r}(\sigma, f)=\int_{\mathrm{GL}_{r}(k) \backslash \mathrm{GL}_{r}\left(\mathbb{A}_{k}\right)} \theta_{r}^{(r)}(h ; f) \mu^{-1}(h) d^{\times} h .
$$

For $* \in\{>,<, \geq, \leq,=\}$, let

$$
\mathrm{GL}_{r}\left(\mathbb{A}_{k}\right)^{* 1}:=\left\{h \in \mathrm{GL}_{r}\left(\mathbb{A}_{k}\right):|\operatorname{det} h|_{\mathbb{A}_{k}} * 1\right\}
$$

Note that

$$
\int_{\mathrm{GL}_{r}(k) \backslash \mathrm{GL}_{r}\left(\mathbb{A}_{k}\right) \leq 1} \theta_{r}^{(r)}(h ; f) \mu^{-1}(h) d^{\times} h
$$

always converges absolutely for every $\sigma$. On the other hand, by Poisson summation formula we get

$$
\theta_{r}(h ; f)=|\operatorname{det} h|_{\mathbb{A}_{k}}^{n} \theta_{r}\left({ }^{t} h^{-1} ; f^{\wedge}\right) .
$$

Hence

$$
\theta_{r}^{(r)}(h ; f)=\sum_{i=0}^{r-1}\left(|\operatorname{det} h|_{\mathbb{A}_{k}}^{n} \theta_{r}^{(i)}\left({ }^{t} h^{-1} ; f^{\wedge}\right)-\theta_{r}^{(i)}(h ; f)\right) .
$$

Let $Q_{r}^{(i)}=\left\{\left(\begin{array}{cc}a_{1} & * \\ 0 & a_{2}\end{array}\right) \in \mathrm{GL}_{r} \mid a_{1} \in \mathrm{GL}_{i}, a_{2} \in \mathrm{GL}_{r-i}\right\}$. It is clear that

$$
\theta_{r}^{(i)}(h ; f)=\sum_{h_{(i)} \in Q_{r}^{(i)}(k) \backslash \mathrm{GL}_{r}(k)}\left(\sum_{x_{i} \in X_{i}^{(i)}(k)} f\left(h^{-1} h_{(i)}^{-1}\left(\begin{array}{c}
x_{i} \\
0
\end{array}\right)\right)\right)
$$

and

$$
\theta_{r}^{(i)}\left({ }^{t} h^{-1} ; f^{\wedge}\right)=\sum_{h_{(i)} \in Q_{r}^{(i)}(k) \backslash \mathrm{GL}_{r}(k)}\left(\sum_{x_{i} \in X_{i}^{(i)}(k)} f\left({ }^{t} h^{t} h_{(i)}\left(\begin{array}{c}
0 \\
x_{i}
\end{array}\right)\right)\right) .
$$

For each $\kappa \in \mathrm{GL}_{r}\left(O_{\mathbb{A}_{k}}\right)$, let $f_{\kappa}(x):=f\left(\kappa^{-1} x\right)$. We then observe that

$$
\begin{gathered}
\int_{\mathrm{GL}_{r}(k) \backslash \mathrm{GL}_{r}\left(\mathbb{A}_{k}\right)>1} \theta_{r}^{(r)}(h ; f) \mu^{-1}(h) d^{\times} h \\
=\int_{\mathrm{GL}_{r}(k) \backslash \mathrm{GL}_{r}\left(\mathbb{A}_{k}\right)>1} \theta_{r}^{(r)}\left({ }^{t} h^{-1} ; f^{\wedge}\right) \mu^{-1}\left({ }^{t} h^{-1}\right) d^{\times} h \\
+\int_{\mathrm{GL}_{r}\left(O_{\mathbb{A}_{k}}\right)} \sum_{i=0}^{r-1} \operatorname{Vol}\left(\operatorname{Mat}_{i \times(r-i)}(k) \backslash \operatorname{Mat}_{i \times(r-i)}\left(\mathbb{A}_{k}\right)\right) \cdot \operatorname{Vol}\left(\mathrm{GL}_{i}(k) \backslash \mathrm{GL}_{i}\left(\mathbb{A}_{k}\right)=1\right) \\
\cdot\left(E_{i}\left(i-\frac{n}{2},\left(f_{\kappa}\right)_{(i, 2)}^{\wedge}\right) \cdot \sum_{\ell=1}^{\infty} q^{\left(-\sigma+\frac{n}{2}-i\right) \ell}\right. \\
\left.-E_{i}\left(i-\frac{n}{2},\left(f_{\kappa}\right)_{(i, 1)}\right) \cdot \sum_{\ell=1}^{\infty} q^{\left(-\sigma-\frac{n}{2}+i\right) \ell}\right) d \kappa .
\end{gathered}
$$


Here for every $f \in S\left(X_{r}\left(\mathbb{A}_{k}\right)\right), f_{(i, 1)}$ and $f_{(i, 2)} \in S\left(X_{i}\left(\mathbb{A}_{k}\right)\right)$ are defined by

$$
f_{(i, 1)}\left(x_{i}\right):=f\left(\begin{array}{c}
x_{i} \\
0
\end{array}\right), \quad f_{(i, 2)}\left(x_{i}\right):=f\left(\begin{array}{c}
0 \\
x_{i}
\end{array}\right)
$$

Therefore when $\sigma>n / 2$,

$$
\begin{aligned}
& \int_{\mathrm{GL}_{r}(k) \backslash \mathrm{GL}_{r}\left(\mathbb{A}_{k}\right)>1} \theta_{r}^{(r)}(h ; f) \mu^{-1}(h) d^{\times} h \\
= & \int_{\mathrm{GL}_{r}(k) \backslash \mathrm{GL}_{r}\left(\mathbb{A}_{k}\right)<1} \theta_{r}^{(r)}\left(h ; f^{\wedge}\right) \mu^{-1}(h) d^{\times} h \\
& +\int_{\mathrm{GL}_{r}\left(O_{\mathbb{A}_{k}}\right)} \sum_{i=0}^{r-1} \operatorname{Vol}\left(\operatorname{Mat}_{i \times(r-i)}(k) \backslash \operatorname{Mat}_{i \times(r-i)}\left(\mathbb{A}_{k}\right)\right) \cdot \operatorname{Vol}\left(\mathrm{GL}_{i}(k) \backslash \mathrm{GL}_{i}\left(\mathbb{A}_{k}\right)^{=1}\right) \\
& \cdot\left(E_{i}\left(i-\frac{n}{2},\left(f_{\kappa}\right)_{(i, 2)}^{\wedge}\right) \cdot \frac{q^{-\sigma+\frac{n}{2}-i}}{1-q^{-\sigma+\frac{n}{2}-i}}+E_{i}\left(i-\frac{n}{2},\left(f_{\kappa}\right)_{(i, 1)}\right) \cdot \frac{1}{1-q^{\sigma+\frac{n}{2}-i}}\right) d \kappa .
\end{aligned}
$$

This gives the meromorphic continuation of $E(\sigma, f)$ (by induction on $r$ ) and (1) holds. In particular, suppose there is a place $v$ of $k$ such that the support of the restriction of $f$ on $X_{r}\left(k_{v}\right)$ is contained in the set of elements with rank $r$ in $X_{r}\left(k_{v}\right)$. Then

$$
\theta_{r}(1, h ; f)=\theta_{r}^{(r)}(1, h ; f)
$$

and

$$
E_{i}\left(i-\frac{n}{2},\left(f_{\kappa}\right)_{i, 1}\right)=0, \quad \forall 0 \leq i \leq r-1
$$

This completes the proof of (3).

Note that

$$
\begin{aligned}
& \int_{\mathrm{GL}_{r}(k) \backslash \mathrm{GL}_{r}\left(\mathbb{A}_{k}\right)=1} \theta_{r}^{(r)}(h ; f) d^{\times} h \\
= & \int_{\mathrm{GL}_{r}(k) \backslash \mathrm{GL}_{r}\left(\mathbb{A}_{k}\right)=1} \theta_{r}^{(r)}\left(h ; f^{\wedge}\right) d^{\times} h \\
& +\int_{\mathrm{GL}_{r}\left(O_{\mathbb{A}_{k}}\right)} \sum_{i=0}^{r-1} \operatorname{Vol}\left(\operatorname{Mat}_{i \times(r-i)}(k) \backslash \operatorname{Mat}_{i \times(r-i)}\left(\mathbb{A}_{k}\right)\right) \cdot \operatorname{Vol}\left(\mathrm{GL}_{i}(k) \backslash \mathrm{GL}_{i}\left(\mathbb{A}_{k}\right)^{=1}\right) \\
& \cdot\left(E_{i}\left(i-\frac{n}{2},\left(f_{\kappa}\right)_{(i, 2)}^{\wedge}\right)-E_{i}\left(i-\frac{n}{2},\left(f_{\kappa}\right)_{(i, 1)}\right)\right) d \kappa .
\end{aligned}
$$

Moreover, from

$$
\left(\begin{array}{c}
x_{i} \\
0
\end{array}\right)=\left(\begin{array}{cc}
0 & I_{i} \\
I_{r-i} & 0
\end{array}\right)\left(\begin{array}{c}
0 \\
x_{i}
\end{array}\right)
$$

we get

$$
\int_{\mathrm{GL}_{r}\left(O_{\mathbb{A}_{k}}\right)} E_{i}\left(i-\frac{n}{2},\left(f_{\kappa}\right)_{(i, 1)}\right) d \kappa=\int_{\mathrm{GL}_{r}\left(O_{\mathbb{A}_{k}}\right)} E_{i}\left(i-\frac{n}{2},\left(f_{\kappa}\right)_{(i, 2)}\right) d \kappa .
$$


Thus by induction on $r$ we have

$$
\begin{aligned}
& E(\sigma, f) \\
= & \int_{\mathrm{GL}_{r}(k) \backslash \mathrm{GL}_{r}\left(\mathbb{A}_{k}\right)^{<1}} \theta_{r}^{(r)}(h ; f)+\theta_{r}^{(r)}\left(h ; f^{\wedge}\right) \mu^{-1}(h) d^{\times} h \\
& +\int_{\mathrm{GL}_{r}(k) \backslash \mathrm{GL}_{r}\left(\mathbb{A}_{k}\right)=1} \theta_{r}^{(r)}(h ; f) d^{\times} h \\
& +\int_{\mathrm{GL}_{r}\left(O_{\mathbb{A}_{k}}\right)} \sum_{i=0}^{r-1} \operatorname{Vol}\left(\operatorname{Mat}_{i \times(r-i)}(k) \backslash \operatorname{Mat}_{i \times(r-i)}\left(\mathbb{A}_{k}\right)\right) \cdot \operatorname{Vol}\left(\mathrm{GL}_{i}(k) \backslash \mathrm{GL}_{i}\left(\mathbb{A}_{k}\right)^{=1}\right) \\
& \cdot\left(E_{i}\left(i-\frac{n}{2},\left(f_{\kappa}\right)_{(i, 2)}^{\wedge}\right) \cdot \frac{q^{-\sigma+\frac{n}{2}-i}}{1-q^{-\sigma+\frac{n}{2}-i}}+E_{i}\left(i-\frac{n}{2},\left(f_{\kappa}\right)_{(i, 1)}\right) \cdot \frac{1}{1-q^{\sigma+\frac{n}{2}-i}}\right) d \kappa . \\
= & \int_{\mathrm{GL}_{r}(k) \backslash \mathrm{GL}_{r}\left(\mathbb{A}_{k}\right)^{<1}} \theta_{r}^{(r)}(h ; f)+\theta_{r}^{(r)}\left(h ; f^{\wedge}\right) \mu^{-1}(h) d^{\times} h \\
& +\int_{\mathrm{GL}_{r}(k) \backslash \mathrm{GL}_{r}\left(\mathbb{A}_{k}\right)=1} \theta_{r}^{(r)}\left(h ; f^{\wedge}\right) d^{\times} h \\
& +\int_{\mathrm{GL}_{r}\left(O_{\mathbb{A}_{k}}\right)} \sum_{i=0}^{r-1} \operatorname{Vol}\left(\operatorname{Mat}_{i \times(r-i)}(k) \backslash \operatorname{Mat}_{i \times(r-i)}\left(\mathbb{A}_{k}\right)\right) \cdot \operatorname{Vol}\left(\mathrm{GL}_{i}(k) \backslash \mathrm{GL}_{i}\left(\mathbb{A}_{k}\right)^{=1}\right) \\
& \cdot\left(E_{i}\left(i-\frac{n}{2},\left(f_{\kappa}\right)_{(i, 1)}^{\wedge}\right) \cdot \frac{1}{1-q^{-\sigma+\frac{n}{2}-i}}+E_{i}\left(i-\frac{n}{2},\left(f_{\kappa}\right)_{(i, 2)}\right) \cdot \frac{q^{\sigma+\frac{n}{2}-i}}{1-q^{\sigma+\frac{n}{2}-i}}\right) d \kappa . \\
= & E\left(-\sigma, f^{\wedge}\right) .
\end{aligned}
$$

Therefore the proof of (2) is complete.

Acknowledgements. The author is grateful to Jing Yu for his steady interest and encouragements. This article was accomplished while the author visited Max Planck Institute for Mathematics. He would like to thank the institution for kindly hospitality and providing a very good working environment. This research was supported in part by National Science Council, and also Max Planck Institute for Mathematics.

\section{REFERENCES}

[1] Bump, D., Automorphic forms and representations, Cambridge studies in advanced mathematics 55 (1997).

[2] Chuang, C.-Y. \& Wei, F.-T., Central critical values of triple product L-functions over global function fields, in preparation.

[3] Chuang, C.-Y. \& Wei, F.-T. \& Yu, J., Rankin-type L-functions over global function fields and Gross-type formula, in preparation.

[4] Haris, S. J., A Siegel formula for orthogonal groups over function fields, Trans. A.M.S. 190 (1974) $223-231$.

[5] Ikeda, T., On the location of poles of the triple L-functions, Compositio math., 83 (1992), 187-237.

[6] Jacquet, H., On the residual spectrum of GL(n), Lecture notes in mathematics 1041 (1984) 185-208.

[7] Jacquet, H. \& Shalika, J., A non-vanishing theorem for zeta functions on GL(n), Invent. math. 38 (1976) $1-16$.

[8] Kudla, S. S., On the local theta correspondence, Invent. math. 83 (1986) 229-255.

[9] Kudla, S. S. \& Rallis, S., On the Weil-Siegel formula, J. Reine Angew. Math. 387 (1988), 1-68.

[10] Kudla, S. S. \& Rallis, S., On the Weil-Siegel formula II, J. Reine Angew. Math. 391 (1988), 65-84.

[11] Lam, T. Y., Introduction to quadratic forms over fields, Graduate Studies in Mathematics vol. 67.

[12] Langlands, R. P., On the functional equation satisfied by Eisenstein series, Lecture notes in mathematics $514(1976)$.

[13] Maass, H., Siegel's modular forms and Dirichlet series, Lecture notes in mathematics 216 (1971).

[14] Moeglin, C. \& Waldspurger, J.-L., Spectral decomposition and Eisenstein series, Cambridge tracts in mathematics 113 (1995). 
[15] Rallis, S., L-functions and the oscillator representation, Lecture notes in mathematics 1245 (1987).

[16] Siegel, C. L., Über die analytische Theorie der quadratischen Formen, Ann. of Math. 36 (1935) 527-606.

[17] Wei, F.-T., On Rankin triple product L-functions over function fields: central critical values, submitted.

[18] Weil, A., Sur certaines groups d'operateurs unitaires, Acta math., 111 (1964) 143-211

[19] Weil, A., Sur la formule de Siegel dans la theorie des groupes classiques, Acta math., 113 (1965) 1-87.

[20] Weil, A., Adeles and Algebraic groups, Progress in Mathematics 23 (1982).

Department of Mathematics, National Tsing-Hua University, Hsinchu 30013, Taiwan

E-mail address: ftwei@mx.nthu.edu.tw 